\title{
Modeling Parameter Space Behavior of Vision Systems Using Bayesian Networks ${ }^{1}$
}

\author{
Sudeep Sarkar and Srikanth Chavali \\ Computer Science and Engineering, University of South Florida, Tampa, Florida \\ E-mail: sarkar@csee.usf.edu; chavali@csee.usf.edu
}

Received September 15, 1998; accepted April 13, 2000

The performance of most vision systems (or subsystems) is significantly dependent on the choice of its various parameters or thresholds. The associated parameter search space is extremely large and nonsmooth; moreover, the optimal choices of the parameters are usually mutually dependent on each other. In this paper we offer a Bayesian network-based probabilistic formalism, which we call the parameter dependence networks (PDNs), to model, abstract, and analyze the parameter space behavior of vision systems. The various algorithm parameters are the nodes of the PDN and are associated with probabilistic beliefs about the optimality of their respective values. The links between the nodes capture the direct dependencies between them and are quantified by conditional belief functions. The PDN structure captures the interdependence among the parameters in a concise and explicit manner. We define information theoretic measures, based on these PDNs, to quantify the global parameter sensitivity and the strength of the interdependence of the parameters. These measures predict the general ease of parameter tuning and performance stability of the system. The PDNs can also be used to stochastically sample the parameter space, to select optimal parameter sets (e.g., in performance evaluation studies), and to choose parameters, given constraints on the choice of some parameters. We also offer a strategy based on stochastic learning automata to generate training data to automatically build these PDNs. The team of learning automata stochastically samples the parameter space in a nonuniform manner with more samples near optimal values. These nonuniform samples are used to infer both the dependency structure and the conditional probabilities of the PDN. We demonstrate the process of construction of the PDN for an isolated vision module with 4 parameters (an edge detector), a coupling of two vision modules with a total of 7 parameters (a small edge grouping module), and a combination of three vision modules with 21 parameters (a complex perceptual organization module). (c) 2000 Academic Press

${ }^{1}$ This work was supported by National Science Foundation Grants IIS-9907141, IRI-9501932, and EIA9729904. 
Key Words: parameter selection; parameter tuning; Bayesian networks; vision systems; learning automaton; vision and learning.

\section{CONTENTS}

\section{Introduction.}

2. Related work.

3. Parameter dependence networks.

4. Stochastic sampling of parameter space. 4.1. Learning automation. 4.2. A team of stochastic automata. 4.3. An illustrating example. 4.4. Convergence. 4.5. Stopping condition. 4.6. Composition of PDN training data.

5. Learning PDN structure and probabilities. 5.1. Structure learning. 5.2. Estimating conditional probabilities.

6. Utility of the parameter dependence networks. 6.1. Generation of parameter sets. 6.2. Constrained selection of parameters. 6.3. Sensitivity of performance to parameter values. 6.4. Strength of interdependence of parameters.

7. Results. 7.1. The vision subsystems and evaluation measures. 7.2. Constructing the PDN. 7.3. Effectiveness of the PDN. 7.4. What can we infer from the PDN?

8. Conclusions.

\section{INTRODUCTION}

Every vision algorithm comes with a set of parameters or thresholds that need to be prespecified. The presence of these parameters is not necessarily a drawback; sometimes they allow us to fine tune the performance of a module to a specific domain, thus imparting flexibility to the process. However, as the number of parameters or thresholds increase, the process of manually choosing the right parameter sets becomes intractable, especially for a network of many vision modules. We can list two main reasons for this computational intractability. First, the search space is extremely large. If $N_{p}$ is the number of parameters to be chosen and $r$ is the number of possible values for each parameter then the total number of possible parameter sets is $r^{N_{p}}$. Second, for a network of vision modules, choice of parameters on a per module basis does not guarantee overall optimal choice. The choice of the parameters might depend upon each other. This can happen not only between parameters of a particular module but also between parameters from different modules. For instance, not only can there be dependence between the choice of the scale, $\sigma$, and the hysteresis thresholds of an edge detector but there can also be dependence between the scale, $\sigma$, and, say, the weight of proximity factor used in a subsequent grouping algorithm. With an increase in edge scale, the contours move away from each other and edges become sparse, which can, in turn, affect the weighting of the proximity factor.

There is a need for a mechanism to abstract the behavior of a vision system over the huge parameter space in a concise and comprehensive manner. This mechanism should model the behavior of the system particularly near optimal parameter values, where optimality is defined in terms of suitable performance measures. It should be able to suggest good parameters at run time. And to ensure adaptability to different situations, we should be able to build the mechanism by learning from examples. We have found that the formalism of Bayesian networks offers an elegant mechanism to satisfy all these goals.

We suggest abstracting the performance of vision systems over the parameter space using a joint probability measure over the different parameter sets. Each possible parameter set 
is associated with a probability measure expressing the belief that it is optimal. Thus, a parameter set with a high belief measure would encode the fact that the parameter set is most likely to enhance the performance of the vision module. There are, however, two major issues with this idea. First, how does one estimate these probability measures? Second, how does one handle the huge dimensionality problem of representing the joint probability density function. It appears as if the dimensionality curse just carries over from the parameter space to the probability space. Fortunately, the formalism of Bayesian networks allows us to handle these problems in an elegant manner.

Bayesian networks are directed acyclic graph representations of joint probability functions where the graph nodes represent the random variables (in our case the parameters) and the links denote direct dependencies between the nodes. The links are quantified by local conditional probabilities. If a set of random variables exhibit a significant amount of conditional independencies, as is usually the case, this directed graph representation is sparse and the conditional probabilities that need to be specified are small in size, resulting in compact representation. Note that the Bayesian network formalism does not require the complete independence assumption. Thus, we do not have to assume that the choices of the parameters are independent of each other. In fact, we explicitly encode the direct dependencies, if any. For expository convenience we call these Bayesian networks over the algorithm parameters the parameter dependence networks or PDNs.

We present a strategy to automatically learn the structure and to estimate the conditional probabilities of a PDN from training data. We generate the training data by stochastically sampling the parameter space and evaluating the performance of a vision algorithm on a given image set with these sampled parameters. The stochastic sampling of the parameter space is not uniform. We use a team of learning automata (LA) in a $N$ player stochastic game framework to selectively sample the parameter space. The team starts off by uniformly sampling the parameter space and gradually concentrating around optimal parameters. Figure 1 depicts this coupling of the PDN and the learning automata in the parameter selection process.

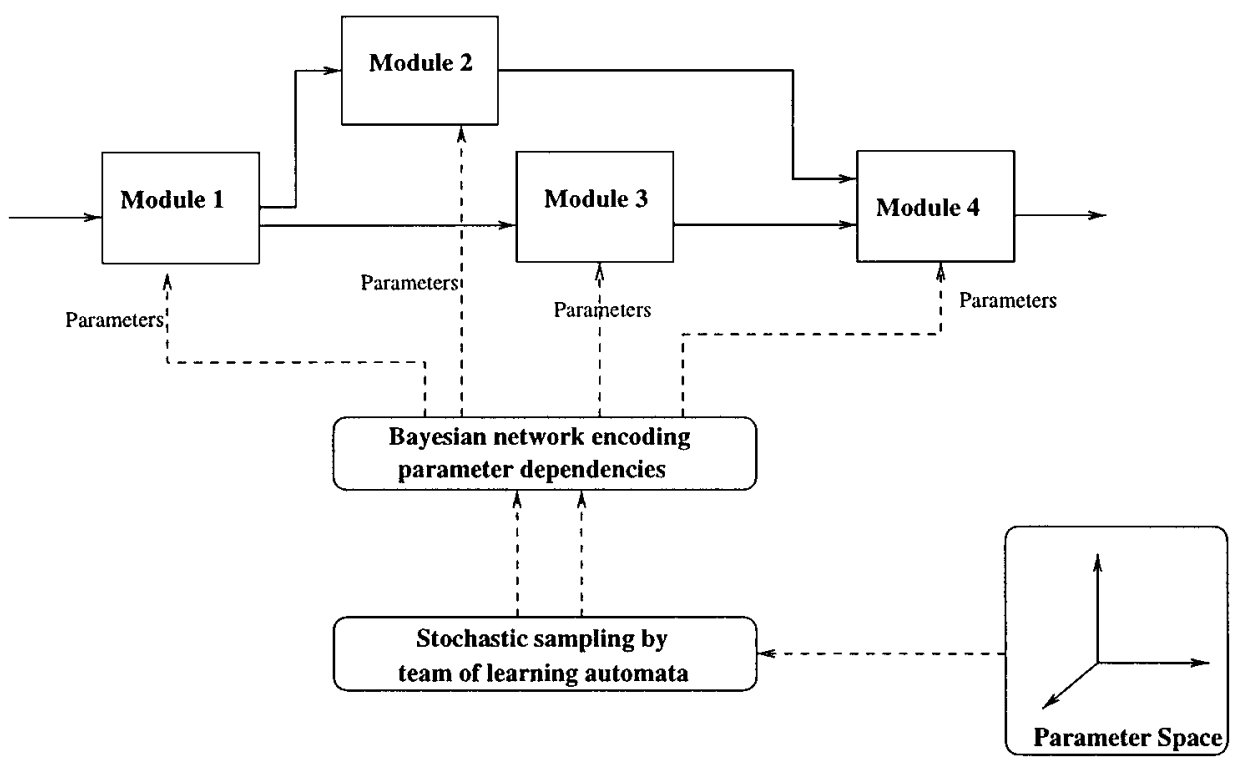

FIG. 1. Coupling of the proposed parameter selection process with vision modules. 
The dependency structure of the PDN offers crucial insight into the nature of the parameter search space. We propose two measures, one based on entropy and the other on mutual information, that capture global parameter sensitivity and the amount of dependence among the parameters. These measures predict the ease of parameter selection of an algorithm and the stability of the performance around optimal choices. Among the other advantages of the PDN is the ability to conditionally select optimal parameters of an algorithm given the values of some of the parameters. This need might arise when certain parameters of an algorithm have to be clamped down to fixed values or to a range of values because of extraneous constraints (e.g., hardware). In this case, we do not have to conduct a parameter search all over again, but we can easily use the PDN to suggest good values for the rest of the parameters, conditioned on the known ones.

Apart from its utility as a stand-alone construct, there are many embedded uses of the proposed parameter dependence networks. First, is, of course, their use in online closedloop feedback-based control of algorithm parameters. Such closed-loop vision systems have been proposed by Murino et al. [1] and Peng and Bhanu [2], among others. The PDNs can be embedded in these frameworks to enable us to tune parameters in an efficient manner by taking into account the interaction among parameters. The second use of the PDNs is in performance evaluation of vision algorithms. One of the important issues in empirical performance characterization is the issue of the choice of the proper algorithm parameters. Before we can compare and contrast algorithms we need to carefully choose the underlying parameters. As we shall discuss in more detail in the next section, the usual practice in performance comparison work has been to choose parameters either based on heuristic manual criteria or by uniformly sampling (or recursively uniformly sampling) the parameter space. It is evident that both these strategies will suffer from the curse of dimensionality as the number of parameters increases.

In the next section we provide a brief overview of the related work in parameter selection in computer vision. We develop the PDN in Section 3, which is followed by a discussion of stochastic sampling using learning automata in Section 4. Section 5 discusses how the PDN is learned from the training data generated by stochastic sampling. Section 6 develops the information theoretic measures and explains how parameter sets can be generated from the PDN. We present results using real vision modules in Section 7 and conclude with Section 8 .

\section{RELATED WORK}

There are two major bodies of work in computer vision that have been deeply concerned with the choice of parameters. One is performance evaluation and characterization, and the other is the closed-loop control of vision systems. In this section, we summarize the different strategies that have been suggested for choosing parameter sets. One of the observations to note from the short review is that either no model of parameter space is used, as in most empirical evaluation studies, or local models are used, as in theoretical performance characterization models. Dependency among parameters is mostly ignored.

There has been progress both in theoretical modeling of performance [3-5] and in empirical performance characterization [6-9]. Ramesh et al. [3] follow a theoretical solution to the problem of parameter selection. They first build random perturbation models [10] for the sequence of vision modules. Statistical data for these perturbation models are then gathered from annotated ground truth data. Theoretical performance measures in terms 
of probabilities of false alarm and detection are derived and optimized to arrive at a set of algorithm parameters for the class of images under consideration. Kanungo et al. [11] present a methodology for performance evaluation where the parameters are chosen based on operating curves. The operating curves relate the probability of misdetection to false alarm. This process of parameter selection is done locally and iteratively; the parameters are fine tuned at each iteration. Forstner [4] proposes selecting parameters by hypothesis testing. An error propagation technique is suggested for deriving these parameters. A significance level for these parameters is specified before the error propagation technique is used. Although methods based on error modeling can offer crucial insights into the behavior of the system, and it is our belief we should opt for it whenever possible, there are practical difficulties in modeling a complex vision system. So far, they have mostly been demonstrated on arrangements of simple vision modules.

The strategies used in empirical evaluation of vision algorithms include manually prespecified parameter sets and variants of exhaustive search strategies. In the comparative study of optical flow algorithms, Barron et al. [8] chose parameters over all the images (fixed parameters) based on manual experimentation. Heath et al. [7] present an edge detector comparison methodology where evaluation of their performance is done by humans. The ranges of the parameters were determined by referring to the papers and experimentations. The number of parameter combinations that were sampled ranged from 6 to 12 for each edge detector. Dougherty and Bowyer [12] present an iterative receiver operating curve (ROC) based methodology for parameter selection in their evaluation of edge detectors. Initially, four values are chosen for each parameter uniformly across the bounds defined for the parameters. A ROC is constructed for these parameters. The parameters are sampled more finely to see if they produce better ROCs. This process is repeated until the change in the area under the curves is less than 5\%. Borra and Sarkar [6] propose a framework for performance characterization of grouping modules where they use fixed and adapted parameters. Based on the performance, quantified using a set of proposed performance measures, on a set of 50 parameter combinations, a subset of 15 parameters was selected. All these strategies fail to scale in a combinatorially graceful manner with the number of parameters.

Houzelle et al. [13] use information about the imaging environment to choose the parameters of a vision system. A set of processes interact through shared data structures in a Prolog framework. Each process has an associated context set that when satisfied causes the process to run. Murino et al. $[1,14]$ suggest a distributed framework for choosing the optimal parameter values of a vision system. Each submodule is associated with a quality evaluation measure and conditional beliefs about the optimality of a module parameter given the parameters of the next and previous modules. To address the large parameter space problem they use qualitative evaluation metrics and coarse Gaussian probability representations, whose choices are heuristic. The PDNs can be embedded in this Murino framework to enable us to tune parameters in a more strict Bayesian framework. Peng and Bhanu [2] present a closed loop recognition system whose segmentation parameters are tuned based on feedback from the recognition module. They employ a team of connectionist Bernoulli quasilinear units with one unit associated with each value that each parameter can take. There is no interaction between the units. Sometimes, the optimal parameter selection process is cast as an optimization problem $[15,16]$. Traditional optimization techniques for parameter search such as hill climbing of gradient descent are then employed. The PDNs can also help such searches by helping to efficiently navigate the parameter space, taking into account the interdependencies of the parameters. 


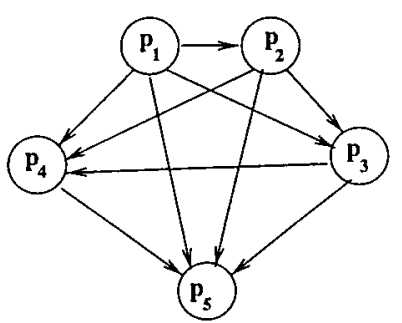

(a)

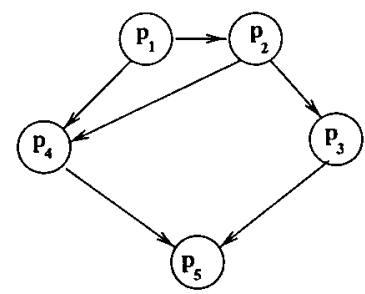

(b)

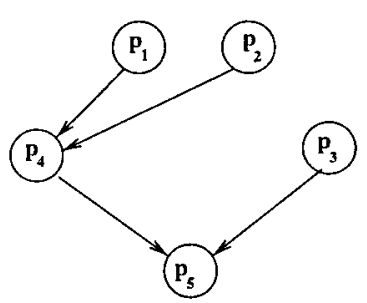

(c)

FIG. 2. Bayesian network representing the joint probability distribution: (a) $P\left(v_{1}, v_{2}, v_{3}, v_{4}, v_{5}\right)=P\left(v_{5}\right.$ | $\left.v_{4}, v_{3}, v_{2}, v_{1}\right) P\left(v_{4} \mid v_{3}, v_{2}, v_{1}\right) P\left(v_{3} \mid v_{2}, v_{1}\right) P\left(v_{2} \mid v_{1}\right) P\left(v_{1}\right)$; (b) $P\left(v_{1}, v_{2}, v_{3}, v_{4}, v_{5}\right)=P\left(v_{5} \mid v_{4}, v_{3}\right) P\left(v_{4} \mid v_{2}, v_{1}\right)$ $P\left(v_{3} \mid v_{2}\right) P\left(v_{2} \mid v_{1}\right) P\left(v_{1}\right)$; (c) $P\left(v_{1}, v_{2}, v_{3}, v_{4}, v_{5}\right)=P\left(v_{5} \mid v_{4}, v_{3}\right) P\left(v_{4} \mid v_{2}, v_{1}\right) P\left(v_{3}\right) P\left(v_{2}\right) P\left(v_{1}\right)$.

\section{PARAMETER DEPENDENCE NETWORKS}

We represent the belief that a particular parameter value is optimal using a probability measure. The notation $P\left(P_{i}=v_{i}\right)$ denotes the belief that the optimal value for the parameter $P_{i}$ is $v_{i}$. Ideally, if we knew that $v_{\text {opt }}$ is the optimal value for parameter $P_{i}$ then $P\left(P_{i}=v_{i}\right)=1$ for $v_{i}=v_{\text {opt }}$ only. ${ }^{2}$ In practice, however, the knowledge of the optimal value of a parameter of a vision algorithm is uncertain; it depends on the particular image under consideration. A probability distribution offers an elegant mechanism for capturing this uncertainty about the optimal value.

The interaction between the optimal choices of two parameters can be captured by the joint probability density, $P\left(P_{1}=v_{1}, P_{2}=v_{2}\right)$. If the choices of the optimal values of the two parameters are independent of each other then, of course, $P\left(P_{1}=v_{1}, P_{2}=v_{2}\right)=P\left(P_{1}=v_{1}\right)$ $P\left(P_{2}=v_{2}\right)$. For $N$ parameters, the joint probability density over the $N$ parameters, $P\left(P_{1}=\right.$ $\left.v_{1}, \ldots, P_{N}=v_{N}\right)$, would capture the dependence among the optimal parameter choices. For notational brevity we will denote the joint probabilities as $P\left(v_{1}, \ldots, v_{N}\right)$. Using the chain rule of conditional probabilities we can express the joint probability density as

$$
P\left(v_{1}, \ldots, v_{N}\right)=P\left(v_{N} \mid v_{N-1}, \ldots, v_{1}\right) P\left(v_{N-1} \mid v_{N-2}, \ldots, v_{1}\right) \cdots P\left(v_{1}\right) .
$$

This factoring of the joint probability density can be depicted in the graphical format shown in Fig. 2a. The nodes of the graph denote the random variables corresponding to the parameters. The links denote direct dependencies and are quantified by the respective conditional probabilities. If some of the parameters or random variables are not directly dependent on each other then the corresponding links would not exist as in Fig. 2b. In the extreme case, all the variables might be independent of each other and the graph would consist of isolated nodes. In practice, however, we neither have full dependence nor do we have complete independence. We usually have partial dependencies with the corresponding graph representation, as shown in Fig. 2b. This graphical representation of a joint probability density is commonly known as a Bayesian network [17].

\footnotetext{
${ }^{2}$ In our definition of a probability measure we take the weak position of allowing subjective estimates. Thus, $P(X=x)$ is really the estimate of the conditional probability that $X=x$ given the present state of knowledge, $P(X=x \mid$ Present Knowledge $)$. The strong position, which is the traditional interpretation, takes a frequency interpretation of probability, i.e., $P(X=x)$ is the limit of the fraction of random outcomes with $X=x$. Of course, probability measures under both the interpretations obey the laws of probability.
} 
Bayesian networks not only provide a visual representation of the underlying dependencies among the random variables but the directed network structure can also act as a computational pathway. Given new evidence about a particular random variable or node, the Bayesian network structure directs the probability updating of all the nodes. Unfortunately it has been shown that updating a general Bayesian network is NP-hard [18]. However, if the Bayesian network structure is a polytree, as it is shown in Fig. 2c, then we can update the probabilities in $\mathcal{O}(N)$ time. (Polytree is a directed tree structure where each node can have multiple parents.)

We define the parameter dependence network as the Bayesian network defined over the algorithm parameters. The conditional probabilities in the network denote the conditional belief in the parameter values being optimal. We set the probability $P\left(v_{1}, \ldots, v_{N}\right)$ to be proportional to the performance of the algorithm, $\beta$, with the parameter values $\left(P_{1}=v_{1}, \ldots, P_{N}=\right.$ $\left.v_{N}\right)$. Thus,

$$
P\left(v_{1}, \ldots, v_{N}\right) \propto \beta\left(P_{1}=v_{1}, \ldots, P_{N}=v_{N}\right) .
$$

Note that the parameter combinations that result in high performance will be assigned high probabilities.

Quantifying performance of a vision algorithm $(\beta)$ is a research topic by itself and in this work we assume that one has a reasonable strategy for it. If a complete vision system is being modeled then the performance, $\beta$, should probably be related to overall measures such as recognition accuracy or execution time, depending on the task requirements. If only a part of the complete vision system is to be modeled then appropriate performance measures should be defined and used, preferably ones that predict behavior of the overall system such as in [6]. If performance cannot be reduced to one scalar measure then one could associate a PDN with each component of the vector performance measures.

\section{STOCHASTIC SAMPLING OF PARAMETER SPACE}

We learn both the structure and the conditional probabilities of the PDN based on sampling of the parameter space. These samples provide information about the nature of the space and the modeled in the PDN as probabilities. An exhaustive deterministic sampling of the space is definitely not practical for any real algorithm with the number of parameters beyond three or four. A completely random uniform sampling is, of course, possible, but this is not the most optimal sampling strategy. The majority of the samples generated by uniform random sampling would most likely be at nonoptimal points. We would like the sampling to be nonuniform with more samples around optimal values so that the PDN can be used to generate near-optimal parameter sets. To solve this problem, we employ a stochastic sampling strategy based on a team of learning automata in a $N$-player game scenario. The team starts with a random sampling of the search space and then asymptotically increases its sampling around the optimal value(s). Thus, the iterative trace of the team very naturally offers us an uneven but effective sampling of the parameter space. The samples capture the performance behavior around the optimal value(s) more than at nonoptimum points, which is exactly what we want the PDN to model. 


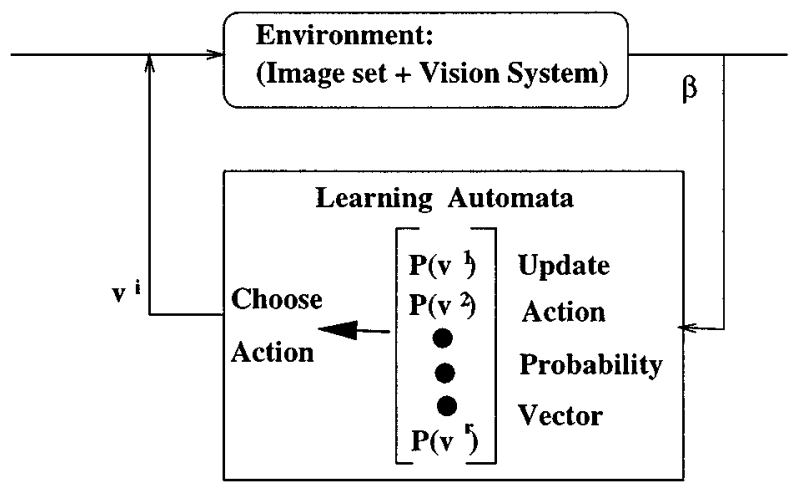

FIG. 3. A simplified model of a learning automaton.

\subsection{Learning Automaton}

The stochastic sampling of the parameter space is achieved using a team of learning automata with one automaton associated with one parameter. A learning automaton is an algorithm that adaptively chooses from a set of possible actions (in the present case, the respective parameter values) on a random environment (the vision algorithm and images) so as to maximize the expected feedback (the performance measure). The reader is referred to [19] for an excellent introduction to learning automata. Figure 3 shows a simple coupling of a learning automaton with the environment. In response to the chosen action, the environment generates a stochastic output $\beta$ that is used by the learning automaton to decide on the next action. The goal of the automaton is to choose the action that results in the maximum expected $\beta$.

A learning automaton decides on the next action by random sampling based on an action probability vector, $\mathbf{p}=\left\{P\left(v^{1}\right), \ldots, P\left(v^{r}\right)\right\}$, defined over the set of actions $\left\{v^{1}, \ldots, v^{r}\right\}$. In the beginning $P\left(v^{1}\right)=\cdots=P\left(v^{r}\right)=1 / r$, signifying that each action is equally likely. Note that these probabilities are different in nature from those defined for the PDN. The probabilities used here are just a mechanism to randomly select an action at each iteration to effect a stochastic search. For notational ease, we will denote $P\left(v^{i}\right)$, the probability of choosing action $v^{i}$, as simply $p_{i}$. On receiving feedback from the environment, the action probability vector is updated using a learning algorithm. The exact nature of the updating algorithm varies. The common strategy, however, is to increase the probability of the action that generates a favorable $\beta$ and decrease the probability of the action that generates an unfavorable feedback. The change in the probabilities are such that $\sum_{i=1}^{r} p_{i}=1$. With each iteration the entropy of the action probability vector decreases until the probability of the optimal action(s) is maximized. It can be shown that the LA will converge if the statistics of the environment are stationary and the updating functions satisfy some minimal conditions. This environmental stationarity assumption implies that the statistics of the image set are stationary or that no new image from a different class $^{3}$ is introduced during the iterations. Note that the LA formulation will allow the introduction of new images in the environment during the learning iterations as long as the stationarity assumption is not violated. The reader is referred to [19] for details about optimality proofs.

\footnotetext{
${ }^{3}$ We define an image class in Section 4.4.
} 


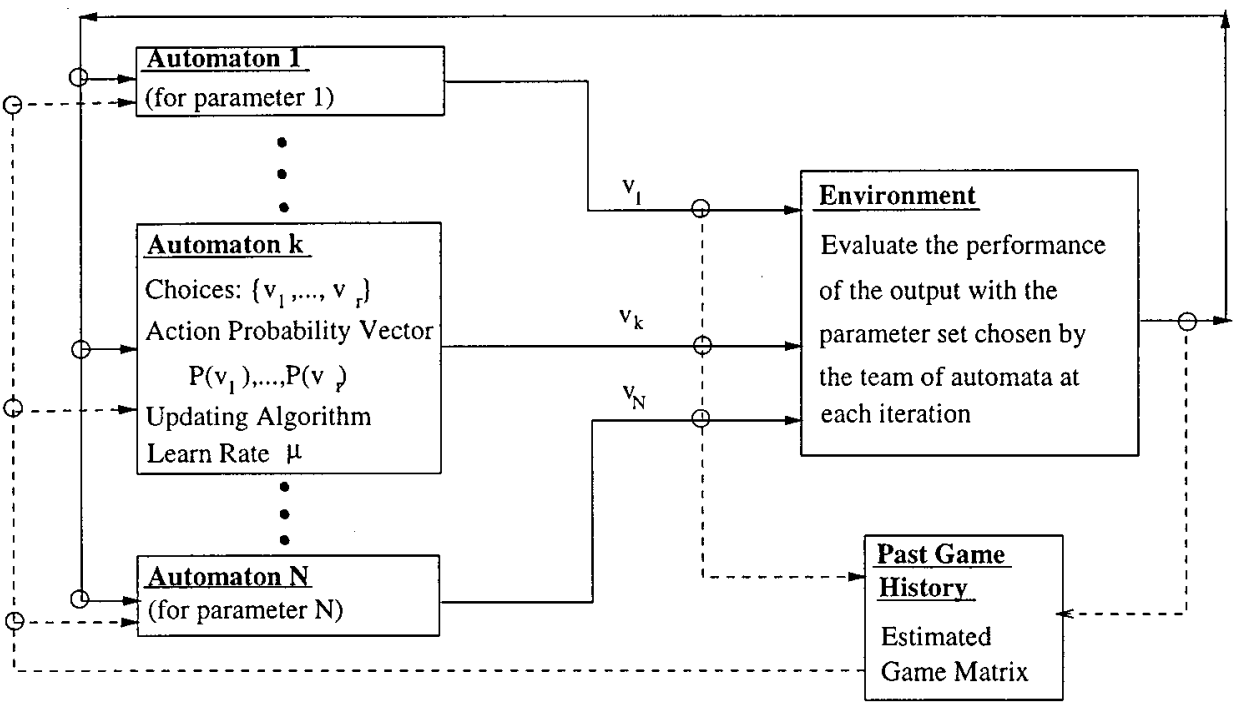

FIG. 4. Equal feedback $N$ player game scenario.

In the present scenario, we associate one learning automaton with one algorithm parameter. The actions correspond to the various values of a parameter. The learning automata, however, do not operate independent of each other, but they work as a team to capture the dependence between the parameters. The probabilistic updating of each automaton takes into account the actions of other automata.

\subsection{A Team of Stochastic Automata}

We map the parameter estimation problem into an $N$-player game by associating with each parameter a player who has to choose from a range of parameter values. We quantize each parameter into $r$ levels ( $r=10$ in our experiments) so that each player has a finite set of moves, plays to make, or actions to choose from. Let us denote this choice set for the $k$ th player to be $\alpha_{k}=\left\{v_{k}^{1}, \ldots, v_{k}^{r}\right\}$. Each player randomly makes a move, which forms part of the chosen parameter set. This parameter set extracts a random reward from the environment, viz. from the network of vision modules and the image set. To generate a reward, the environment applies the network of modules, with the parameter set, on a subset of training images and computes the average performance. The performance can be quantified in terms of the quality of the task outcome. We do not concern ourselves with how this is achieved. Any reasonable approach should suffice. The quantified performance is returned as a reward to each player. Based on this common feedback, $\beta$, each player chooses its next move. The objective of each player is to choose an action so as to maximize this feedback over time (see Fig. 4).

The strategy is to maintain estimates of the expected feedback for every combination of moves made as a multidimensional matrix called the (estimated) game matrix $\hat{\mathbf{D}}=\left\{\hat{D}_{i_{1}, \ldots, i_{N}}\right\}{ }^{4}$ Recall that the $i_{k}$ th action of the $k$ th automaton is denoted by $v_{k}^{i_{k}}$. Then, 
$\hat{D}_{i_{1}, \ldots, i_{N}}$ stores the average reward for the play $\left\{v_{1}^{i_{1}}, \ldots, v_{N}^{i_{N}}\right\} .{ }^{5}$ The estimated game matrix $\hat{\mathbf{D}}$ is really an approximation of the underlying game matrix governing the game $\mathbf{D}=\left\{D_{i_{1}}, \ldots, i_{N}\right\}$, which is composed of the expected feedbacks for every combination of moves, $\left\{i_{1}, \ldots, i_{N}\right\}$,

$$
D_{i_{1}, \ldots, i_{N}}=E\left(\beta \mid \alpha_{1}=v_{1}^{i_{1}}, \ldots, \alpha_{N}=v_{N}^{i_{N}}\right),
$$

where $v_{k}^{i_{k}}$ is the $i_{k}$ th action of the $k$ th automaton. We, of course, do not know the game matrix a priori. If the statistics of this game matrix, however, are stationary then algorithms can be designed based on its estimates so that the game converges to the global optimum.

In our case, this environmental stationarity assumption implies that no new image from a different class is introduced during the iterations. This condition is satisfied if the training image set is predetermined and fixed.

Each player keeps a projection of the global game matrix and updates its action probability vector based on it. This projection represents the maximum expected feedback received for its various actions. Let $E_{k}^{j}$ denote the maximum expected reward to the $k$ th player when it plays $v_{k}^{j}$. We denote the vector composed of these individual rewards by $\mathbf{E}_{\mathbf{k}}=\left\{E_{k}^{j} \mid\right.$ $j=1, \ldots, r\}$ and call it the individual game vector. This individual game vector can be looked upon as a projection of the game matrix. Thus,

$$
E_{k}^{j}=\max _{\left\{i_{s}, s \neq k\right\}} D_{i_{1}, \ldots, i_{k-1}, j, i_{k+1}, \ldots, i_{N}} .
$$

The term $\left\{i_{s}, s \neq k\right\}$ denotes the set of possible combinations of moves of all the players except for the $k$ th player. Let the globally optimal play for the $k$ th player be $v_{k}^{m_{k}}$; then

$$
\max _{\left\{i_{s}\right\}} D_{i_{1}, \ldots, i_{N}}=\max _{j} E_{k}^{j}=E_{k}^{m_{k}} \quad \text { for all } k=1, \ldots, N .
$$

The term $\left\{i_{s}\right\}$ denotes the set of possible combinations of moves of all the players. So, each player can reach the globally optimum point by choosing plays according the individual game vector $\mathbf{E}_{\mathbf{k}}=\left\{E_{k}^{j}\right\}$. Of course, in practice we only have an estimate of this vector, $\hat{\mathbf{E}}_{\mathbf{k}}$, which is computed from the estimated game matrix $\hat{D}_{i_{1}, \ldots, i_{N}}$.

$$
\hat{E}_{k}^{j}=\max _{\left\{i_{s}, s \neq k\right\}} \hat{D}_{i_{1}, \ldots, i_{k-1}, j, i_{k+1}, \ldots, i_{N}}
$$

Based on the environment feedback, $\beta$, and the individual game vector estimate, $\hat{E}_{k}^{j}$, each automaton updates its action probabilities. Let us denote the iteration number by $n$. We will denote the value of each variable at the $n$th iteration by appending $n$ as an argument to the symbols. Thus, $\beta(n)$ denotes the environmental feedback at the $n$th iterations and $\alpha_{k}(n)$ denotes the play of the $k$ th automaton at the $n$th iteration. Let $\left\{v_{1}^{i_{1}}, \ldots, v_{k}^{i_{k}}, \ldots, v_{N}^{i_{N}}\right\}$ be the play of the $N$ automata at the $n$th iteration. The action probability vector for the $k$ th automaton is updated as follows [20].

Figure 5 shows the sequence of updating steps and the flow of information. First, the action probability vector is updated using the individual game matrix estimate, $\hat{\mathbf{E}}_{\mathbf{k}}(n)$, constructed so far. Recall that $\hat{\mathbf{E}}_{\mathbf{k}}(n)$ stores the maximum feedback received for each action for the $k$ th automaton. Thus, $\hat{E}_{k}^{i_{k}}(n)$ is the maximum feedback received for the action $\alpha_{k}(n)=v_{k}^{i_{k}}$,

\footnotetext{
${ }^{5}$ It might appear that we would require a significant amount of memory to store this game matrix estimate. In practice, however, this estimated game matrix is sparse and can be efficiently stored. The number of nonzero entries will be at most equal to the number of iterations, which is typically far less than the maximum possible size of the matrix.
} 


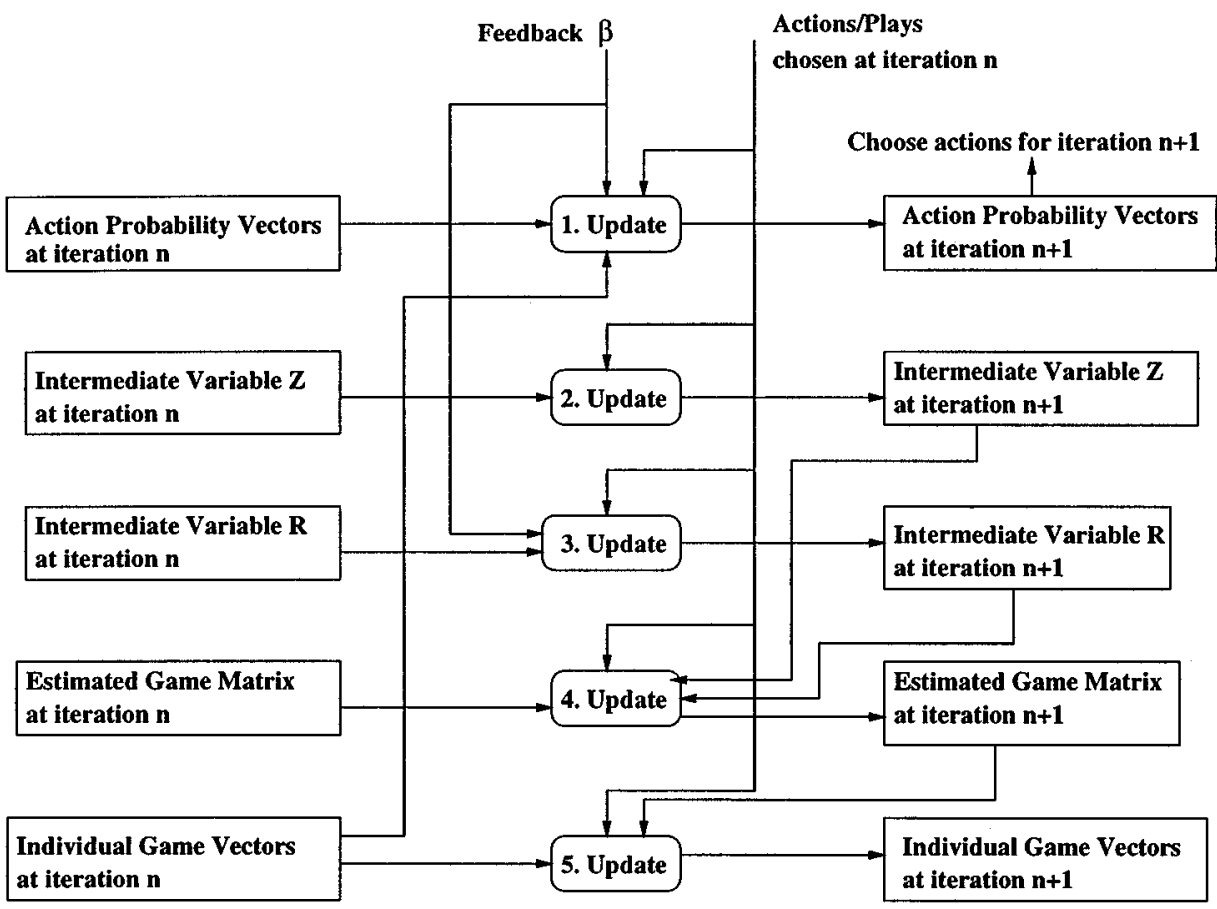

FIG. 5. Sequencing and flow of information in the LA team updating process at each iteration.

the action chosen at the $n$th iteration. The probabilities of all actions with individual game estimates larger than the estimate for the current action are increased and those that are lower are decreased. Thus, for actions, $v_{k}^{j}$, that have lesser maximum feedback than that for the current action, $v_{k}^{i_{k}}$, or $\hat{E}_{k}^{j}(n)<\hat{E}_{k}^{i_{k}}(n)$, we lower their probabilities as follows.

$$
p_{k}^{j}(n+1)=p_{k}^{j}(n)-\mu\left(\hat{E}_{k}^{i_{k}}-\hat{E}_{k}^{j}\right) p_{k}^{j}(n) \frac{p_{k}^{i_{k}}(n)}{r-1}
$$

For actions, $v_{k}^{j}$, that have greater maximum feedback than that for the current action, $v_{k}^{i_{k}}$, or $\hat{E}_{k}^{j}(n)>\hat{E}_{k}^{i_{k}}(n)$, we increase their probabilities as follows.

$$
p_{k}^{j}(n+1)=p_{k}^{j}(n)+\mu\left(\hat{E}_{k}^{j}-\hat{E}_{k}^{i_{k}}\right)\left(1-p_{k}^{j}(n)\right) \frac{p_{k}^{i_{k}}(n)}{r-1}
$$

Probability for the action chosen at the current iteration, $v_{i_{k}}^{k}$, is updated by simply normalizing as follows.

$$
p_{k}^{i_{k}}(n+1)=1-\sum_{j \neq i_{k}} p_{k}^{j}(n+1)
$$

This normalization might result in either an increase or a decrease of the action probability depending on how high or low $\hat{E}_{k}^{i_{k}}$ is compared to the other $\hat{E}_{k}^{j}$ 's.

Note that in the above probability updating the change is proportional to (i) the learn rate $\mu$, (ii) the difference in the individual maximum feedbacks $\hat{E}_{k}^{i_{k}}-\hat{E}_{k}^{j}$, and (iii) the current action probabilities. Thus, the amount of change will depend on the iteration. There would be faster movement at later iterations when the action probability vector converges 
to definite actions and the difference between the maximum and the minimum probabilities increases. The nature of the search space also affects the rate of convergence. If the search space includes large changes between its maximum and minimum points then $\hat{E}_{k}^{i_{k}}-\hat{E}_{k}^{j}$ would tend to be high and the action probability will change faster than for a bland search space with small change in its relief.

The next steps at each iteration consist of updating the total game matrix and the individual game vector estimates, $\hat{E}_{k}^{j}(n+1)$. We use two intermediate variables $R$ and $Z$ to compute the game matrix estimate, $\hat{D}_{j_{1}, \ldots, j_{N}}(n+1)$, which, in turn, determines $\hat{E}_{k}^{j}(n+1)$. The variables $R$ and $Z$ have the same dimensions as the estimated game matrix. The total feedback for each move combination until the current iteration is stored in $R$ and the total number of times a particular move combination was chosen by the automata team is stored in $Z$. The estimated game matrix is then given by just the (nonzero) entry-wise division of $R$ by $Z$. Note that at each step we need to update only one entry of $R, Z$, and $\hat{D}$, namely, the entry corresponding to the plays at the $n$th iteration, $\left\{v_{1}^{i_{1}}, \ldots, v_{N}^{i_{N}}\right\}$. Then, using the notation that $X_{i, \ldots, j}$ denotes the entry corresponding to the play $\left\{v_{1}^{i}, \ldots, v_{N}^{j}\right\}$, where $X$ is one of $\hat{D}, Z$, or $R$, the updates are:

$$
\begin{aligned}
& R_{i_{1}, \ldots, i_{N}}(n+1)=R_{i_{1}, \ldots, i_{N}}(n)+\beta(n) \\
& Z_{i_{1}, \ldots, i_{N}}(n+1)=Z_{i_{1}, \ldots, i_{N}}(n)+1 \\
& \hat{D}_{i_{1}, \ldots, i_{N}}(n+1)=\frac{R_{i_{1}, \ldots, i_{N}}(n+1)}{Z_{i_{1}, \ldots, i_{N}}(n+1)} .
\end{aligned}
$$

As the last step, given the updated game matrix, we update the individual game vectors, $\mathbf{E}_{\mathbf{k}}$, which store the maximum feedback for each move on a per player basis. Like the game matrix, we need to update only one entry of the individual game vector of each automaton, i.e., the entry corresponding to the play made by that automaton. Thus, the $k$ th automaton updates its entry as follows:

$$
\hat{E}_{k}^{i_{k}}(n+1)=\max \left\{\hat{E}_{i_{k}}^{k}(n), \hat{D}_{i_{1}, \ldots, i_{N}}(n+1)\right\} .
$$

\subsection{An Illustrating Example}

Consider a simple three-parameter problem, where each parameter can take two values, 0 or 1 , with a total number of parameter combinations being just eight. We need three learning automata, each of which has two possible moves. Let the actual performance of this vision algorithm for each parameter combination for a class of images be a random variable with uniform distribution specified in Table 1 , where $U(a, b)$ denotes a uniform distribution between $a$ and $b$.

TABLE 1

The Actual Game Matrix Underlying the Example Game

\begin{tabular}{cccccccc}
\hline$(0,0,0)$ & $(0,0,1)$ & $(0,1,0)$ & $(0,1,1)$ & $(1,0,0)$ & $(1,0,1)$ & $(1,1,0)$ & $(1,1,1)$ \\
$U(0.1,0.3)$ & $U(0.0,0.2)$ & $U(0.2,0.4)$ & $U(0.3,0.5)$ & $U(0.5,0.7)$ & $U(0.6,0.8)$ & $U(0.7,0.9)$ & $U(0.0,0.2)$ \\
\hline
\end{tabular}

Note. The first row lists all the possible eight move combinations of three players, each with two possible actions. The second rows lists the uniform probability density functions governing the feedback randomness for each possible move combination. 
In the beginning, the action probability vectors of the three learning automata are all uniform. The total game matrix and the individual game vector entries are all zero.

Player 1: $\quad \hat{\mathbf{E}}_{\mathbf{1}}(0)=[0.00,0.00] \quad \mathbf{p}_{\mathbf{1}}(0)=[0.5,0.5]$

Player 2: $\quad \hat{\mathbf{E}}_{\mathbf{2}}(0)=[0.00,0.00] \quad \mathbf{p}_{\mathbf{2}}(0)=[0.5,0.5]$

Player 3: $\quad \hat{\mathbf{E}}_{\mathbf{3}}(0)=[0.00,0.00] \quad \mathbf{p}_{\mathbf{3}}(0)=[0.5,0.5]$

Indices $(0,0,0) \quad(0,0,1) \quad(0,1,0) \quad(0,1,1) \quad(1,0,0) \quad(1,0,1) \quad(1,1,0) \quad(1,1,1)$

$\begin{array}{lllllllll}\mathrm{R} & 0.00 & 0.00 & 0.00 & 0.00 & 0.00 & 0.00 & 0.00 & 0.00 \\ \mathrm{Z} & 0 & 0 & 0 & 0 & 0 & 0 & 0 & 0 \\ \hat{D} & 0.00 & 0.00 & 0.00 & 0.00 & 0.00 & 0.00 & 0.00 & 0.00\end{array}$

Based on the initial action probability vectors, let the three automata randomly choose the actions $\left(\alpha_{1}(1)=1, \alpha_{2}(1)=0, \alpha_{3}(1)=0\right)$, which solicits a random feedback of, say, 0.53 . On receiving this feedback the variables of each automaton and the whole game are updated as follows. First, the action probability vectors are updated based on the feedback received and the old individual game vector estimates. For the first iteration, when all the individual game vectors are zero, there is basically no update of the action probability vectors. Next, the total game matrix is updated via the intermediate variables $Z$ and $R$ that store the total feedback and count, respectively, for each combination of actions. Thus, in the first iteration the entries at index $(1,0,0)$ are updated. Last, based on the estimated game matrix, $\hat{D}$, the individual game vectors are updated. Again, note that for each automaton only one entry corresponding to the play made is updated. At the end of first iteration, the variables are as follows:

Player 1: $\quad \hat{\mathbf{E}}_{\mathbf{1}}(1)=[0.00,0.53] \quad \mathbf{p}_{\mathbf{1}}(1)=[0.5,0.5]$

Player 2: $\quad \hat{\mathbf{E}}_{\mathbf{2}}(1)=[0.53,0.00] \quad \mathbf{p}_{\mathbf{2}}(1)=[0.5,0.5]$

Player 3: $\quad \hat{\mathbf{E}}_{\mathbf{3}}(1)=[0.53,0.00] \quad \mathbf{p}_{\mathbf{3}}(1)=[0.5,0.5]$
Indices
$(0,0,0)$
$(0,0,1)$
$(0,1,0)$
$(0,1,1)$
$(1,0,0)$
$(1,0,1)$
$(1,1,0) \quad(1,1,1)$

$\begin{array}{lllllllll}\mathrm{R} & 0.00 & 0.00 & 0.00 & 0.00 & 0.53 & 0.00 & 0.00 & 0.00 \\ \mathrm{Z} & 0 & 0 & 0 & 0 & 1 & 0 & 0 & 0 \\ \hat{D} & 0.00 & 0.00 & 0.00 & 0.00 & 0.53 & 0.00 & 0.00 & 0.00\end{array}$

Let the random actions chosen for the second iteration be $\left(\alpha_{1}(2)=1, \alpha_{2}(2)=1, \alpha_{3}(2)=0\right)$, which solicits a random feedback of 0.75 . The updated variables are as follows:

Player 1: $\quad \hat{\mathbf{E}}_{\mathbf{1}}(2)=[0.00,0.75] \quad \mathbf{p}_{\mathbf{1}}(2)=[0.47,0.53]$

Player 2: $\quad \hat{\mathbf{E}}_{\mathbf{2}}(2)=[0.53,0.75] \quad \mathbf{p}_{\mathbf{2}}(2)=[0.51,0.49]$

Player 3: $\quad \hat{\mathbf{E}}_{\mathbf{3}}(2)=[0.75,0.00] \quad \mathbf{p}_{\mathbf{3}}(2)=[0.53,0.47]$

Indices $\quad(0,0,0) \quad(0,0,1) \quad(0,1,0) \quad(0,1,1) \quad(1,0,0) \quad(1,0,1) \quad(1,1,0) \quad(1,1,1)$

$\begin{array}{lllllllll}\mathrm{R} & 0.00 & 0.00 & 0.00 & 0.00 & 0.53 & 0.00 & 0.75 & 0.00 \\ \mathrm{Z} & 0 & 0 & 0 & 0 & 1 & 0 & 1 & 0 \\ \hat{D} & 0.00 & 0.00 & 0.00 & 0.00 & 0.53 & 0.00 & 0.75 & 0.00\end{array}$

Note that the action probability vectors are updated so as to reflect bias toward actions with larger entries in the individual game vectors at the last iteration. Thus, for the second player $\alpha_{2}=0$ is preferred reflecting the bias in $\hat{\mathbf{E}}_{\mathbf{2}}(1)$. Since so far in the game the second player has chosen both the possible plays, it is the one with two nonzero entries in the individual 
game vector. And, for the first and the third players the entries in the individual game vectors corresponding to the only action chosen so far are updated. The total game matrix estimate has two nonzero entries corresponding to the two play combinations chosen.

For the third iteration, let the chosen random action be $\left(\alpha_{1}(3)=1, \alpha_{2}(3)=1, \alpha_{3}(3)=1\right)$, which solicits a random feedback of 0.02 . The updated variables are as follows:

$\begin{array}{lll}\text { Player 1: } & \hat{\mathbf{E}}_{\mathbf{1}}(3)=[0.00,0.75] & \mathbf{p}_{\mathbf{1}}(3)=[0.44,0.56] \\ \text { Player 2: } & \hat{\mathbf{E}}_{\mathbf{2}}(3)=[0.53,0.75] & \mathbf{p}_{\mathbf{2}}(3)=[0.50,0.50] \\ \text { Player 3: } & \hat{\mathbf{E}}_{\mathbf{3}}(3)=[0.75,0.02] & \mathbf{p}_{\mathbf{3}}(3)=[0.54,0.46]\end{array}$
Indices
$(0,0,0)$
$(0,0,1)$
$(0,1,0)$
$(0,1,1)$
$(1,0,0)$
$(1,0,1)$
$(1,1,0) \quad(1,1,1)$

$\begin{array}{lllllllll}\mathrm{R} & 0.00 & 0.00 & 0.00 & 0.00 & 0.53 & 0.00 & 0.75 & 0.02 \\ \mathrm{Z} & 0 & 0 & 0 & 0 & 1 & 0 & 1 & 1 \\ \hat{D} & 0.00 & 0.00 & 0.00 & 0.00 & 0.53 & 0.00 & 0.75 & 0.02\end{array}$

Note that for the second player, the bias that was slightly tilted toward $\alpha_{2}=0$ at last iteration is now reversed based on the updated individual game vector $\hat{\mathbf{E}}_{\mathbf{2}}(2)$. Also note that the individual game vectors for the first and the second player did not change at this iteration since the feedback received, 0.02 , is less than the corresponding entries in the vectors.

For the fourth iteration, let $\left(\alpha_{1}(4)=0, \alpha_{2}(4)=0, \alpha_{3}(4)=1\right)$ solicit a random feedback of 0.16 . The updated variables are as follows:

$\begin{array}{lll}\text { Player 1: } & \hat{\mathbf{E}}_{\mathbf{1}}(4)=[0.16,0.75] & \mathbf{p}_{\mathbf{1}}(4)=[0.42,0.58] \\ \text { Player 2: } & \hat{\mathbf{E}}_{\mathbf{2}}(4)=[0.53,0.75] & \mathbf{p}_{\mathbf{2}}(4)=[0.50,0.50] \\ \text { Player 3: } & \hat{\mathbf{E}}_{\mathbf{3}}(4)=[0.75,0.16] & \mathbf{p}_{\mathbf{3}}(4)=[0.56,0.44]\end{array}$

Indices $\quad(0,0,0) \quad(0,0,1) \quad(0,1,0) \quad(0,1,1) \quad(1,0,0) \quad(1,0,1) \quad(1,1,0) \quad(1,1,1)$

$\begin{array}{lllllllll}\mathrm{R} & 0.00 & 0.16 & 0.00 & 0.00 & 0.53 & 0.00 & 0.75 & 0.02 \\ \mathrm{Z} & 0 & 1 & 0 & 0 & 1 & 0 & 1 & 1 \\ \hat{D} & 0.00 & 0.16 & 0.00 & 0.00 & 0.53 & 0.00 & 0.75 & 0.02\end{array}$

All the entries of the individual game vectors are now filled with some feedback from the environment. The action probability vectors continue to reflect the same bias as in the last iteration. And, we have four nonzero entries in the game matrix estimate corresponding to the four iterations.

The process continues. The trace of the learning automata variables for the first 20 iterations of a sample run is shown in Table 2. At the end of these iterations, the mode of the action probability vector is for the play $(1,1,0)$, which is the optimal combination. In fact, the optimal play combination tends to be chosen more than any other combination after the first 25 iterations. Also, notice how the estimated game matrix is converging to the mean of the stochastic process governing the feedback.

\subsection{Convergence}

The team of automata essentially effects a stochastic search, with the process gradually honing onto optimal values. The rate of convergence is governed by not only a user supplied learn rate, $\mu$, but also by the nature of search space. What is particularly attractive is that the process is guaranteed to converge to the optimal value (see Thathachar and Shastry [20]). The caveat is that we have to use the right choice of the learning parameter $\mu$. There are no underlying assumptions of "smoothness" of the entries in the game matrix, only that 


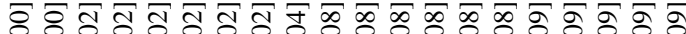

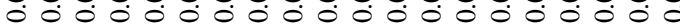

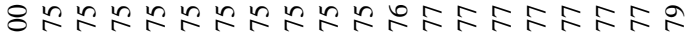
- 0000000000000000000

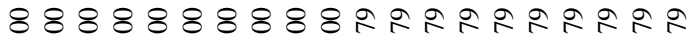
00000000000000000000

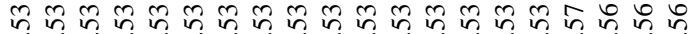

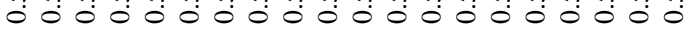
8.8888.88888888888 0.000 .000 .000

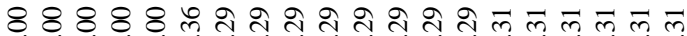

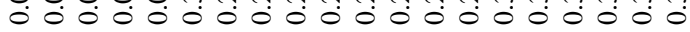
\&\&\& $0 \infty \infty \infty \infty \infty \infty \infty \infty \infty \infty \infty \infty \infty \infty \infty$ ०0 0 0 :

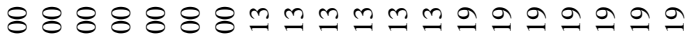

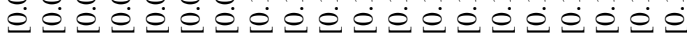

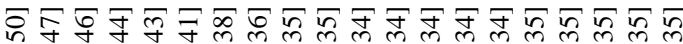

2 0000000000000000000

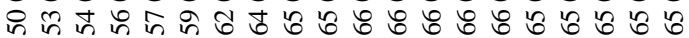

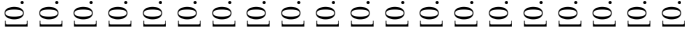

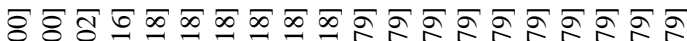

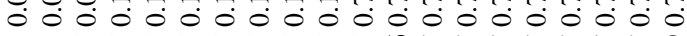

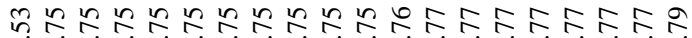
$0 \dot{0} \dot{0} \dot{0} \dot{0} \dot{0} \dot{0} \dot{0} \dot{0} \dot{0} \dot{0} \dot{0} \dot{0} \dot{0} \dot{0} 0 \dot{0}$

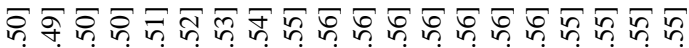
00000000000000000000

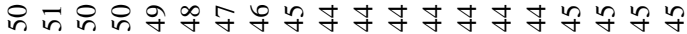
00000000000000000000

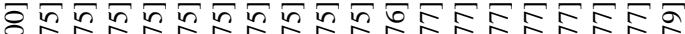

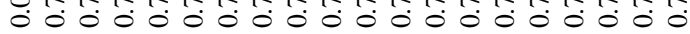

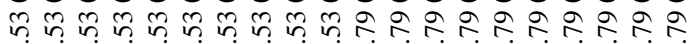
- 000000000000000000

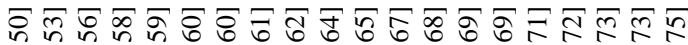

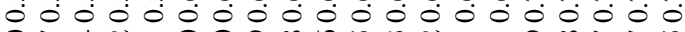

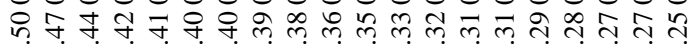
00000000000000000000

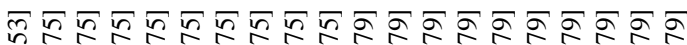

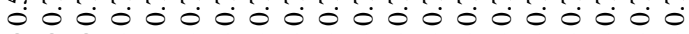
\& \& \& 0 -

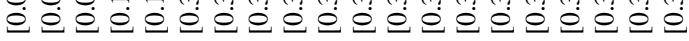

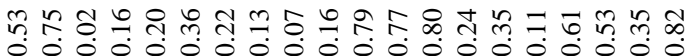

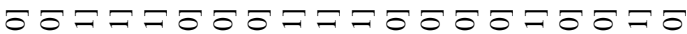
$0-100-10=10-10-100 月-1$

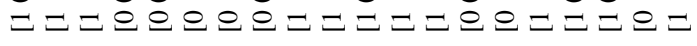


the matrix entries be stationary. This stationarity condition translates to the stationarity of performance of the vision algorithm over the iterations. This condition will be satisfied if no new image from a different class, as defined below, is introduced during the iterations, ${ }^{6}$ which is satisfied if the training image set is predetermined and closed.

We define two images to be from the same class if the performance of the vision algorithm under study is similar on these images for the same parameters. Images in the same class would tend to have low variance in performance. For most vision algorithms, images from the same domain would tend to be from the same class.

There is additional advantage in insisting that the images be from the same class. If images from different classes are present in the training set, then the performance variance would tend to be high and consequently the differences in mean performance for different parameter combinations would tend to be low. This would translate into small differences between peaks and valleys in the estimated game matrix and the individual game vectors. Consequently, the convergence would be slow. Recall that the changes in the action probability vectors are dependent on three factors, one of which is the variation in the individual game vector entries. Thus, if the training set of images are from the same class then the convergence would be fast.

The rate of convergence is also inversely related to $\mu$. If one chooses a very small $\mu$ then the learning algorithm is very slow but the probability of finding the global optimum increases. A large $\mu$ implies faster convergence but does not guarantee a global optimal point. In our experiments, we have found that changing $\mu$ with iteration gives us good results. We start with $\mu=0$ for the first 100 iterations to let the algorithm form a starting estimate of the game matrix. For later iterations, $\mu$ is set to 0.1 . Although convergence to the optimal value is desirable, it is not necessary for constructing the PDN. All we need are samples close to optimal values, which the learning automata team guarantees to us. Figure 6 illustrates this converging behavior of the samples for a three-player game where each player has 10 possible moves. The underlying random game matrix has a maximum expected value at the move $(5,5,5)$. The learn rate was 0.05 . Figures $6 \mathrm{a}$ through $6 \mathrm{~d}$ show the sampling of the space in units of 100 iterations. Notice that the sampling is uniform toward the beginning and in about 300 iterations, the sampling has concentrated around the optimal value.

\subsection{Stopping Condition}

The commonly used stopping condition is expressed in terms of the probabilities in the action probability vectors. For example, one possibility is to stop when the maximum probability exceeds a certain threshold, e.g., 0.9. The optimal action is the one with the highest probability. In our case, we are not too much concerned about reaching the exact optimal value. We are just interested in exploring the space around the optimal value(s); hence, we are able to stop earlier. Note that the action probabilities are updated based on the estimated individual game vectors, $\mathbf{E}_{\mathbf{k}}$, and these individual game vectors are (maximum) projections of the total game matrix. A change in $\mathbf{E}_{\mathbf{k}}$ signifies that the LA team has found a new potential peak in the total game matrix and the action probability updates are changed accordingly, depending on the strength of the new peak. If $\mathbf{E}_{\mathbf{k}}$ does not change then the

\footnotetext{
${ }^{6}$ Note that the LA formulation does allow the possibility of introducing new images as the iterations progress, making it compatible for online applications, if needed. However, in our present usage this issue is not germane.
} 


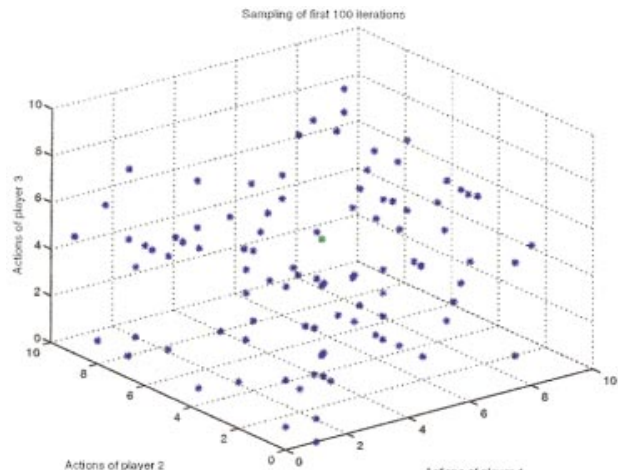

(a)

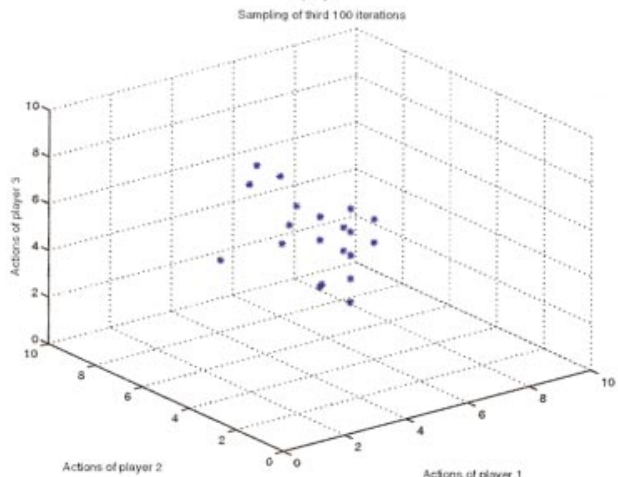

(c)

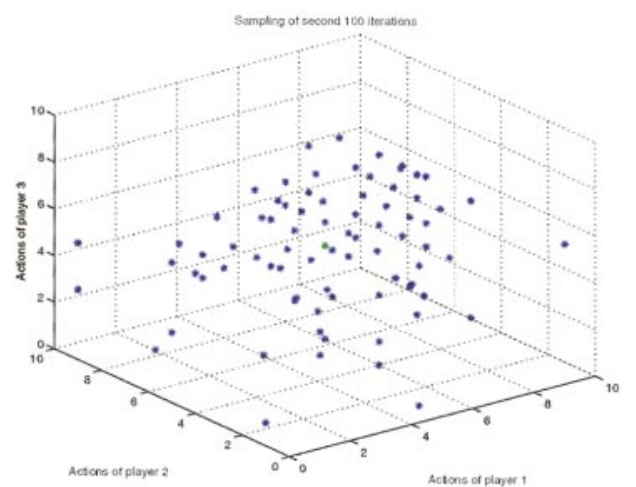

(b)

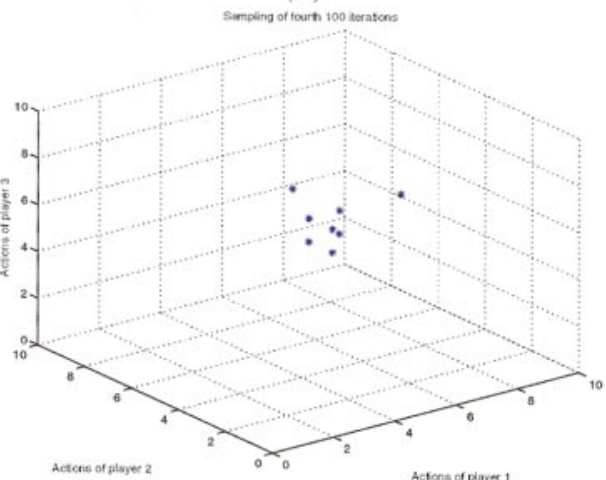

(d)

FIG. 6. Sampling of the search space by three automata with 10 actions each in (a) the first 100 iterations, (b) the second 100 iterations, (c) the third 100 iterations, and (d) the fourth 100 iterations.

trend of the change of the action probability vectors remain the same: the probabilities for actions with large $E_{k}^{j}$ will increase and those with low values will decrease. Thus, a static $\mathbf{E}_{\mathbf{k}}$ over a number of iterations, say 200, signifies that no new peak has been detected in the game matrix and this forms our stopping condition. We stop when there are no changes in any of the individual game vectors $\mathbf{E}_{\mathbf{k}}$ s for, say, 200 iterations.

\subsection{Composition of PDN Training Data}

The training data that are used to construct a PDN consist of a table of parameter combinations, along with a performance measure representing the goodness of the combination. This goodness can be defined in terms of the average performance across all the images in the training set or on individual images. We prefer the second option since the learned PDN would then implicitly encode the image to optimal parameter mapping. And consequently, the PDN will have the ability to suggest good parameters on a per-image basis rather than parameters that result in good average performance across the image class. However, if the latter case is desirable in some circumstances, we would choose parameter combinations from the learning automata trace that perform well across the images.

The performance of a parameter combination on each individual image is available since at each learning iteration the vision algorithm is executed on a set of images to compute the average performance, which is fed back to the learning automata team. From 
this data, for each image we select $N_{P}$ parameter combinations that resulted in the best performance. Thus, the training data set consists of $N_{I} N_{P}$ parameter combinations along with their performance on a per-image basis, where $N_{I}$ denotes the number of images in the LA environment. Note that since the learning automata team operates to maximize the performance across the image set in an average sense, the best parameter combinations selected for a particular image will not, in general, result in extremely poor performance on other images.

\section{LEARNING PDN STRUCTURE AND PROBABILITIES}

The task is to automatically build a PDN from training data composed of the LA iterative trace. As discussed, the training data are essentially composed from the second and the third columns of Table 2. The PDN learning problem is similar to learning Bayesian networks from examples, which has been studied extensively (see [21] for a recent survey). The essential difference is that usual training data for learning Bayesian networks consist of just state assignments of nodes (some of which might be missing), whereas in our case, we have state assignments along with probability estimates. There are two aspects to learning Bayesian networks: structure inference and estimation of associated probabilities. Bayesian network structuring algorithms include those based on maximum likelihood principles [22], minimum cross-entropy [23], minimum description length (MDL) [24], Akaike information criteria (AIC), and the Bayesian information criteria (BIC) [25]. The strategies for learning the conditional probabilities of the Bayesian network include approaches based on the principle of maximum likelihood (ML) [26], maximum a posterior probability (MAP) [27], and incremental learning [28].

In our present work, we have adopted the undirected polytree structure learning methodology of Chow and Liu [23], later adapted for directed acyclic structured Bayesian networks by Rebane and Pearl [17,29]. This algorithm results in an optimal polytree structure for the Bayesian network; i.e., there are no cycles in the underlying undirected graph. Possibilities of other sophisticated structure learning methodologies will be the subject of future exploration. For the vision modules that we have studied empirically, we have found the polytree Bayesian network structure to be sufficient. The imposition of a polytree structure on the PDN is convenient from a computational standpoint. It is possible to update probabilities in such structures in polynomial time. Bayesian updating for a general Bayesian network is NP-hard [18].

The following discussion of the polytree algorithm for structure learning is a summary of the approach with the essential modifications mentioned along the way. The unique nature of our training data and the imprecision of the probability estimates led us to constrain the tree structure and to reformulate some of the crisp test conditions suggested by Rebane and Pearl. We also provide implementation details of the method, which are usually not mentioned in theoretical Bayesian network structuring work but are, nevertheless, important in practice.

\subsection{Structure Learning}

One way of estimating the optimal directed polytree structure is to first estimate the optimal underlying undirected tree structure and then to infer the link directions. The optimal undirected tree structure that approximates the joint probability density can be found by 
optimizing the Kubiak-Lebback (KL) mutual information distance measure [23]. Let $P^{\prime}(\mathbf{p})$ denote the probability density represented by the undirected tree structured network defined on the parameters, $\mathbf{p}=\left\{P_{1}, \ldots, P_{N}\right\}$. If $P(\mathbf{p})$ is the underlying joint probability density, the $\mathrm{KL}$ distance measure is

$$
K L\left(P, P^{\prime}\right)=\sum P(\mathbf{p}) \log \frac{P(\mathbf{p})}{P^{\prime}(\mathbf{p})} .
$$

Chow and Liu [23] have shown that the optimal undirected tree structure is the maximum spanning tree of the graph of pairwise mutual information whose nodes are the random variables and the links are weighted by the mutual information between the two variables, $I\left(P_{i}, P_{j}\right)$.

$$
I\left(P_{i}, P_{j}\right)=\sum P\left(v_{i}, v_{j}\right) \log \frac{P\left(v_{i}, v_{j}\right)}{P\left(v_{i}\right) P\left(v_{j}\right)}
$$

This mutual information measure is nonnegative and is zero when the two parameters, $P_{i}$ and $P_{j}$, are independent of each other. Thus, the maximum spanning tree of the mutual information graph gives us the set of edges that connect the most strongly dependent nodes. Rebane and Pearl $[17,29]$ have shown that this spanning tree captures all conditional independencies among random variable triples and is optimal under the constraint of maintaining a tree structure.

For the example described in Section 4.3, the pairwise mutual information based on the first 200 iterations are: $I\left(P_{1}, P_{2}\right)=0.008, I\left(P_{2}, P_{3}\right)=0.143$, and $I\left(P_{1}, P_{3}\right)=0.004$. (For simplicity and without loss of generality, we considered the raw first 200 learning automata trace and did not use the strategy described in Section 4.6 for this example.) To compute these mutual pieces of information, we first computed the pairwise-joint and singleton probability over the parameters, the information for which is available in the iteration trace data. For instance, $P\left(v_{1}, v_{2}\right)=\sum_{v_{3}} \beta\left(P_{1}=v_{1}, P_{2}=v_{2}, P_{3}=v_{3}\right) / \sum_{v_{1}, v_{2}, v_{3}} \beta\left(P_{1}=v_{1}, P_{2}=v_{2}\right.$, $\left.P_{3}=v_{3}\right)$, which we can compute from data in the iteration trace. The maximum spanning tree based on the computed mutual information is given by $P_{1}-P_{2}-P_{3}$.

5.1.1. Constrained maximum spanning tree. In the maximum spanning tree construction stage we impose an extra constraint that the maximum number of neighbors of a node be $K$. This value of $K$ determines the maximum number of parents a node can have in the (directed) Bayesian network. And, the number of parents determines the size of the conditional probability density that needs to be specified. If the number of parents of a node is $K$, then the total number of conditional probability mass functions that need to be estimated is $r^{K}$ (all the possible states of the parents), where $r$ is the number of states of each parent. Each conditional probability function, in turn, has $r$ states. Note that these conditional probabilities would have to be estimated from a finite set of training data. The finiteness of the training data effects the accuracy of the probability estimates. The uncertainty of the probability estimates increases with decreased training data because all the possible states of the parents might not be present in the training data a sufficient number of times. This uncertainty can actually be quantified by the relative entropy between the actual distribution and its empirical distribution. If we fix the acceptable uncertainty of the probability estimates then we can restrict the number of parents and thus decide on $K$, as shown below. 
Abe [30] has recently shown that "the expected relative entropy (or the expected divergence) between the finite probability distribution $Q$ on $\{1,2, \ldots, l\}$ and its empirical one obtained from the sample of size $N$ drawn from $Q$ is $\ldots$ asymptotically $(l-1) \frac{\log (e)}{2 N}$ which is independent of $Q$." In our case we would like to compute the expected divergence between the estimated conditional probabilities and the actual underlying probability. The conditional probability of a node $X$ given the states of its parents $\Pi(X)$ can be expressed as a ratio of two joint probabilities using the Bayes theorem,

$$
P(X=x \mid \Pi(X)=\pi(X))=\frac{P(X=x, \Pi(X)=\pi(X))}{P(\Pi(X)=\pi(X))},
$$

where $x$ and $\pi(X)$ are the states of the node, $X$, and its parents, $\Pi(X)$, respectively. The joint probability in the numerator is over a larger number of variables and its uncertainty dominates the uncertainty of the conditional probability. So, we concentrate on estimating the divergence of the estimate of $P(X=x, \Pi(X)=\pi(X))$ from the actual underlying joint probability using Abe's theorem mentioned above. For $K$ parents and $r$ possible states of each node, we have $r^{K+1}$ states for the joint probability. Thus the expected relative entropy, $E(S)$, is given by

$$
E(S)=\left(r^{K+1}-1\right) \frac{\log e}{2 N},
$$

where $N$ is the size of the training data. We compare this relative entropy with the maximum possible entropy of the joint probability distribution over $r^{K+1}$ states, which is $\log \left(r^{K+1}\right)$ (for a uniform distribution). We choose $K$ such that the expected relative divergence of the estimated probability from the actual one is less than the maximum possible entropy of the actual one. Thus, we choose $K$ such that

$$
\left(r^{K+1}-1\right) \frac{\log e}{2 N} \leq(K+1) \log r .
$$

In our experiments, $r=10$ and $N$ is typically 2000 to 3000 , hence, $K \leq 3$ or 4 satisfies the above equation. So we restrict the maximum number of neighbors of nodes in the spanning tree to be 3 or 4 .

5.1.2. Inferring link directions. To complete the structure specification of the PDN we have to assign directions to the links. These directions would capture not only direct dependencies between variables but also the conditional dependences. It can be shown that a Bayesian network is a minimal independence map over all possible triples of variables [17]. Thus, it is possible to assign link directions to the undirected graph by iteratively resolving the direction over triples of random variables.

Consider the triple structures in Fig. 7. Figure 7a captures the fact that the parents are independent of each other; however, they become dependent on each other once we know the state of the child, i.e., $P\left(v_{i}, v_{j} \mid v_{k}\right) \neq P\left(v_{i} \mid v_{k}\right) P\left(v_{j} \mid v_{k}\right)$ but $P\left(v_{i}, v_{j}\right)=P\left(v_{i}\right) P\left(v_{j}\right)$. On the other hand the structures in Figs. 7b-7d capture the fact that the nodes $P_{i}$ and $P_{j}$ are independent of each other given the state of $P_{k} ; P_{k}$ separates $P_{i}$ and $P_{j}$. Thus, $P\left(v_{i}, v_{j} \mid\right.$ $\left.v_{k}\right)=P\left(v_{i} \mid v_{k}\right) P\left(v_{j} \mid v_{k}\right)$ in Fig. 7b. For Figs. 7c and 7d too, this conditional independence holds, which can be seen by observing that for Fig. 7c, $P\left(v_{i} \mid v_{k}, v_{j}\right)=P\left(v_{i} \mid v_{k}\right)$ and for Fig. 7d, $P\left(v_{j} \mid v_{k}, v_{i}\right)=P\left(v_{j} \mid v_{k}\right)$. It is difficult to uniquely choose among the structures in Figs. $7 b-7 d$ just based on statistical data. As Pearl [17] points out, we need external 


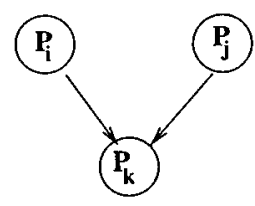

(a)

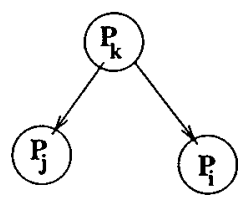

(b)

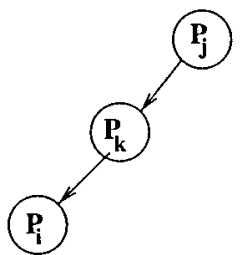

(c)

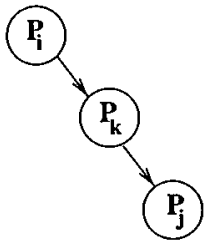

(d)

FIG. 7. All possible dependency link directions over a tree structured network over a triple of random variables, $P_{i}, P_{j}$, and $P_{k}$.

knowledge, such as causality among the variables, to decide among the three structures. Based on just the mutual information estimate from statistical data, it is possible to separate only the structure in Fig. 7a from those in Figs. 7b-7d.

Since the mutual information between two variables quantifies the dependence of one variable upon another, it is easy to see that for Fig. 7a we should have

$$
I\left(P_{i}, P_{j}\right)=0, \quad \text { and } I\left(P_{i}, P_{j} \mid P_{k}\right)>0,
$$

where $I\left(P_{i}, P_{j}\right)$ is given by Eq. (13) and

$$
I\left(P_{i}, P_{j} \mid P_{k}\right)=\sum P\left(v_{i}, v_{j}, v_{k}\right) \log \frac{P\left(v_{i}, v_{j} \mid v_{k}\right)}{P\left(v_{i} \mid v_{k}\right) P\left(v_{j} \mid v_{k}\right)} .
$$

For the structures in Figs. 7b-7d we will have the complimentary case of Eq. (17).

$$
I\left(P_{i}, P_{j}\right)>0, \quad \text { and } I\left(P_{i}, P_{j} \mid P_{k}\right)=0
$$

In practice, however, the strict equality in Eqs. (17) and (19) will never be satisfied because we do not have knowledge of the exact mutual information. All we have are just estimates. Hence, we replace the above conditions by the following.

If $\left.\left(I\left(P_{i}, P_{j}\right) \mid P_{k}\right)-I\left(P_{i}, P_{j}\right)\right)>0$ then we have the structure $\left(P_{i} \rightarrow P_{k} \leftarrow P_{j}\right)$ or we have one of the following three structures: $\left(P_{i} \leftarrow P_{k} \rightarrow P_{j}\right),\left(P_{i} \rightarrow P_{k} \rightarrow P_{j}\right)$, or $\left(P_{i} \leftarrow P_{k} \leftarrow P_{j}\right)$.

We resolve among $\left(P_{i} \leftarrow P_{k} \rightarrow P_{j}\right),\left(P_{i} \rightarrow P_{k} \rightarrow P_{j}\right)$, or $\left(P_{i} \leftarrow P_{k} \leftarrow P_{j}\right)$ by favoring the structure that results in the least number of parents for a node in the final network. Recall that at each node of the Bayesian network we store the conditional probability of the node given the possible states of the parents. The representational space complexity of the conditional probability grows exponentially with the number of parents. Thus, a final Bayesian network with a low number of parents for each node is desirable.

We iteratively apply the above test (Eq. (20)) to all triple combinations of parents and children of the spanning tree. We start with the triple with the maximum value for $\left(I\left(P_{i}, P_{j} \mid\right.\right.$ $\left.\left.P_{k}\right)-I\left(P_{i}, P_{j}\right)\right)$ and proceed to consider lower ones (in decreasing order) at later iterations. At each iteration, we set the link directions of the links that are undirected between the three nodes being considered at that iteration. If a link direction is assigned at one iteration, it is not changed at later ones. Thus, the triple with large value for $\left(I\left(P_{i}, P_{j} \mid P_{k}\right)-I\left(P_{i}, P_{j}\right)\right)$, 
in some sense, constrains the assignment of directions for other links. If at some stage there is more than one choice for a link direction then, as we mentioned before, we favor the direction that results in a lesser number of parents for the nodes.

For the example described in Section 4.3, as we saw earlier, the minimum spanning tree was given by $P_{1}-P_{2}-P_{3}$. The mutual information, $I\left(P_{1}, P_{3}\right)$, is 0.004 and the conditional mutual information, $I\left(P_{1}, P_{3} \mid P_{2}\right)$, is 0.026 . Thus, $\left(I\left(P_{1}, P_{3} \mid P_{2}\right)-I\left(P_{1}, P_{3}\right)\right)>0$ and the directed link structure is $\left(P_{1} \rightarrow P_{2} \leftarrow P_{3}\right)$. That is, $P_{1}$ and $P_{3}$ are independent but are conditionally dependent given information about $P_{2}$.

\subsection{Estimating Conditional Probabilities}

The next step is to learn the conditional probabilities of the PDN from the training samples. There are various strategies for estimating these probabilities. We choose the simplest one, namely, that of relative frequency, which can be shown to be the maximum likelihood estimate of the probability [21]. Other sophisticated techniques for estimating the probabilities exist and they can be the subject of future work.

Recall that we would like the probabilities in the PDN to reflect the belief in the optimality of a parameter combination i.e., $P\left(v_{1}, \ldots, v_{N}\right) \propto \beta\left(v_{1}, \ldots, v_{N}\right)$ where $\beta$ is the scalar performance measure. To estimate the conditional probability of a node $X$, given the states of its parents, $\Pi(X)=\pi(X)$, we sum the $\beta$ 's over all cases when $X=x$ and $\Pi(X)=\pi(X)$ and normalize it. Thus, we have

$$
P(x \mid \pi(X))=\frac{\sum_{\text {Cases with } X=x, \Pi(X)=\pi(X)} \beta\left(v_{1}, \ldots, v_{N}\right)}{\sum_{\text {Cases with } \Pi(X)=\pi(X)} \beta\left(v_{1}, \ldots, v_{N}\right)} .
$$

For the example described in Section 4.3, whose PDN structure we found to be $\left(P_{1} \rightarrow\right.$ $\left.P_{2} \leftarrow P_{3}\right)$, probabilities that need to be computed are $P\left(v_{1}\right), P\left(v_{3}\right)$, and the conditional probability, $P\left(v_{2} \mid v_{1}, v_{3}\right)$. Based on the first 200 iterations of the LA team, the estimates of these probabilities are

$$
\begin{aligned}
P\left(v_{1}\right) & =[0.05,0.95] \\
P\left(v_{2} \mid P_{1}=0, P_{3}=0\right) & =[0.26,0.74] \\
P\left(v_{2} \mid P_{1}=0, P_{3}=1\right) & =[0.17,0.83] \\
P\left(v_{2} \mid P_{1}=1, P_{3}=0\right) & =[0.34,0.66] \\
P\left(v_{2} \mid P_{1}=1, P_{3}=1\right) & =[0.87,0.13] \\
P\left(v_{3}\right) & =[0.73,0.27] .
\end{aligned}
$$

Note that, based on the conditional probability structure, the state $(1,1,0)$ is the most likely one (probability $=0.46$ ), which is the parameter combination with the best performance.

\section{UTILITY OF THE PARAMETER DEPENDENCE NETWORKS}

The PDN essentially models the behavior of an algorithm around optimal values in a comprehensive manner. This model has many uses and can provide crucial insights into the behavior of a network of vision modules. 


\subsection{Generation of Parameter Sets}

One of the obvious uses of PDN is the generation of good parameter sets that enhance algorithm performance. There are two possible vision tasks that can benefit from this. First, is performance evaluation of vision algorithms. To compare and contrast algorithms, one has to properly choose the various algorithm parameters. The PDN can be used to suggest good parameter sets for a vision algorithm in a rigorous manner. The strategy would be to learn the PDN based on a training set and then to evaluate the algorithms on a different image set using parameters generated by the PDN. The second task where the parameter generation capability of the PDN would be beneficial is online parameter tuning of vision systems or modules. Closed-loop vision systems try to adapt parameters to a domain. The PDN can assist such closed-loop strategies by offering a mechanism to efficiently navigate the parameter space by generating a sequence of random parameter sets guided by the PDN. The PDN guided sampling should yield faster adaptation than completely random sampling or local search-based strategies.

Parameter sets can be easily generated by Gibbs stochastic simulation based on the PDN $[17,31]$. The first iteration starts off with a random assignment of values to the parameters in the PDN. Then at successive iterations, the value of a parameter, $P_{i}$, is assigned randomly, conditioned on the values of the parameters that are its neighbors in the PDN. Each iteration involves two phases: local computation followed by random generation of state. In the first phase, the conditional probabilities of the states of a parameter node given the current states of its neighbors are computed. In the second phase, a value is randomly assigned to each parameter node based on these conditional probabilities. All nodes are processed sequentially in this manner and the process repeats itself at the next iteration. This procedure generates parameter sets whose statistics are governed by the probabilities of the PDN. Figure 8 shows a parameter node, $P_{i}$, along with its neighbors. Let us denote the parents of any node, $X$, by $\Pi(X)$ and their states by $\pi(x)$. Also, let the children of the node $P_{i}$ be $Y_{1}, \ldots, Y_{M}$. Let all the other nodes of the Bayesian network, except the parents and children

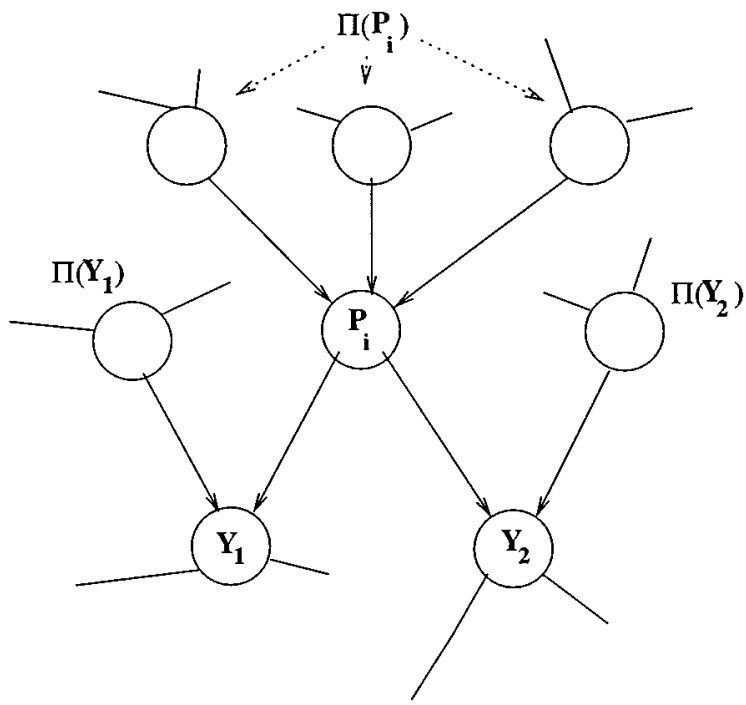

FIG. 8. Context of a node $P_{i}$ in a parameter dependence network. 
of $P_{i}$, be denoted by $\Omega\left(P_{i}\right)$ taking on states, $\omega\left(P_{i}\right)$. Then the conditional probability of the node $P_{i}$ given the states of all the nodes of the tree can be expressed as:

$$
P\left(v_{i} \mid \pi\left(P_{i}\right), y_{i}, \ldots, y_{M}, \omega\left(P_{i}\right)\right) .
$$

Using Bayes' theorem, this conditional probability can be expressed as

$$
P\left(v_{i} \mid \pi\left(P_{i}\right), y_{i}, \ldots, y_{M}, \omega\left(P_{i}\right)\right)=\frac{P\left(v_{i}, y_{i}, \ldots, y_{M}, \omega\left(P_{i}\right) \mid \pi\left(P_{i}\right)\right)}{P\left(y_{i}, \ldots, y_{M}, \omega\left(P_{i}\right)\right)} .
$$

Based on the conditional probability factoring depicted in the PDN shown in Fig. 8, we can further simplify the above expression.

$$
P\left(v_{i} \mid \pi\left(P_{i}\right), y_{i}, \ldots, y_{M}, \omega\left(P_{i}\right)\right)=\frac{P\left(v_{i} \mid \pi\left(P_{i}\right)\right) P\left(y_{1} \mid \pi\left(Y_{1}\right)\right) \cdots P\left(y_{M} \mid \pi\left(Y_{M}\right)\right)}{P\left(y_{i}, \ldots, y_{M}, \omega\left(P_{i}\right)\right)}
$$

The denominator $P\left(y_{i}, \ldots, y_{M}, \omega\left(P_{i}\right)\right)$ is a constant across all states, $v_{i}$, and can be ignored in the computation stage and easily accounted for by normalizing the conditional probabilities over all the states, $v_{i}$. Thus, the conditional probability of a node given the states of all the other nodes in the network is proportional to the product of the local conditional probabilities in the PDN. Thus,

$$
P\left(v_{i} \mid \pi\left(P_{i}\right), y_{i}, \ldots, y_{M}, \omega\left(P_{i}\right)\right) \propto P\left(v_{i} \mid \pi\left(P_{i}\right)\right) P\left(y_{1} \mid \pi\left(Y_{1}\right)\right) \cdots P\left(y_{M} \mid \pi\left(Y_{M}\right)\right) .
$$

For the example described in Section 4.3, whose PDN structure we computed to be $\left(P_{1} \rightarrow\right.$ $P_{2} \leftarrow P_{3}$ ), this implies that conditional probabilities of each node can be computed using

$$
\begin{aligned}
& P\left(v_{1} \mid v_{2}, v_{3}\right) \propto P\left(v_{1}\right) P\left(v_{2} \mid v_{1}, v_{3}\right) \\
& P\left(v_{2} \mid v_{1}, v_{3}\right) \propto P\left(v_{2} \mid v_{1}, v_{3}\right) \\
& P\left(v_{3} \mid v_{1}, v_{3}\right) \propto P\left(v_{3}\right) P\left(v_{2} \mid v_{1}, v_{3}\right)
\end{aligned}
$$

This implies that based on the current state of the neighbors we can compute the (proportional) estimates of $P\left(v_{i} \mid \pi\left(P_{i}\right), y_{i}, \ldots, y_{M}, \omega\left(P_{i}\right)\right)$ for all possible $v_{i}$. We can easily factor in the priors by normalizing the estimated probability vector. Based on the normalized probability vector, we assign the next value of the parameter node $P_{i}$. At each iteration, we repeat this process for all the parameter nodes. As iterations proceed, the effect of a parameter is propagated across the network. The samples at later iterations reflect the stationary probabilities of the parameters.

\subsection{Constrained Selection of Parameters}

For some applications, external considerations, such as hardware or time considerations, might dictate constraints on the range of values for some parameters. In such cases, we would like to choose the values for other parameters so that performance is maximized. This problem can be easily addressed if the PDN is available. The parameter nodes whose values are constrained would be clamped down to the specified values, and values for other parameters would be generated by Gibbs sampling. If a specific value is known for a parameter then it would not be allowed to change at each iteration of Gibbs sampling. 
Otherwise, if a range of values is known for a node then the parameter would be allowed to vary only over that range at each iteration. Thus, given the PDN, such a constrained choice of parameters can be easily made.

\subsection{Sensitivity of Performance to Parameter Values}

Sensitivity of an algorithm to parameter values is a crucial measure, especially for real time performance. The precise choice of the values for certain parameters may be important for good performance. On the other hand, certain parameters might not need to be fine tuned precisely. The most common strategy for quantifying parameter sensitivity is to consider perturbations around an operating point. The idea is to consider the extent of change in performance if a parameter value is perturbed by a small amount. This is a local measure. The alternative approach, which we adopt, is to consider a more global measure.

The probabilistic notion used in defining the PDN offers us a way of considering global context in formulating a sensitivity measure. Recall that $P\left(P_{i}=v_{i}\right)$ captures the belief that $v_{i}$ is optimal and is quantified by the average algorithm performance with that parameter value. If the performance of an algorithm is very high for one particular parameter value and very low for others then the associated probability function would have one very high peak. If the performance of an algorithm does not vary with different parameter values then $P\left(P_{i}=v_{i}\right)$ would be more or less uniform. This difference in the nature of the distribution can be captured by the entropy, $S\left(P_{i}\right)$, of the probability function, which is equal to $-\sum_{v_{i}} P\left(P_{i}=v_{i}\right) \log P\left(P_{i}=v_{i}\right)$. The entropy measure, $S\left(P_{i}\right)$, captures the uncertainty associated with the probability distribution. If the optimal value is unique and very distinct from the rest of the values, $S\left(P_{i}\right)$ would be low. If all different values for the parameter result in similar performance then $S\left(P_{i}\right)$ would be high.

If instead of computing the entropy for singleton probability densities we consider the joint probability space over all the parameters then we will get a global picture of the nature of the performance variation that takes into account interparameter interactions. We thus suggest the following entropic measure as a global way of quantifying parameter sensitivity.

$$
S\left(P_{1}, \ldots, P_{N}\right)=-\sum_{v_{1}, \ldots, v_{N}} P\left(v_{1}, \ldots, v_{N}\right) \log P\left(v_{1}, \ldots, v_{N}\right)
$$

We compute this global entropic measure using local computations on the PDN. Let a parameter node, $P_{i}$, have parents denoted by $\Pi\left(P_{i}\right)$ with their possible values denoted by $\pi\left(P_{i}\right)$. Using the conditioned factored representation of the joint probability function, we can easily show that

$$
S\left(P_{1}, \ldots, P_{N}\right)=-\sum_{i}\left(\sum_{v_{i}, \pi\left(P_{i}\right)} P\left(v_{i}, \pi\left(P_{i}\right)\right) \log P\left(v_{i} \mid \pi\left(P_{i}\right)\right)\right) .
$$

The ouside (first) sum on the right-hand side of Eq. (29) is over the parameter nodes of the PDN. The inside (second) sum is over the states of the local neighborhood of the node and can be computed locally at each node. The conditional probability $P\left(P_{i}=v_{i} \mid \Pi\left(P_{i}\right)=\right.$ $\left.\pi\left(P_{i}\right)\right)$ is present a priori at the node and the local joint probability $P\left(P_{i}=v_{i}, \Pi\left(P_{i}\right)=\pi\left(P_{i}\right)\right)$ can be estimated by locally accumulating count during Gibbs sampling. Thus, the entropic sensitivity measure can be estimated by local computations on the PDN; this is 
computationally more efficient than summing over a potentially large joint probability space.

\subsection{Strength of Interdependence of Parameters}

The strength of interdependence of parameters is an important aspect of an algorithm. If all the parameters are independent of each other then it implies that the optimal parameter choices can be made independently and change in choice of the value of a parameter does not affect the optimal choices of other parameters. If the parameters, however, are strongly dependent on each other then optimal choices of parameters cannot be chosen independent of each other. Choice of one parameter should take into account choices of the other parameters.

We suggest a global measure of the parameter interdependence, $I\left(P_{1}, \ldots, P_{N}\right)$ based on the KL distance between two probability functions (see Section 5.1) and of the form similar to the mutual information measures in Eqs. (13) and (18).

$$
I\left(P_{1}, \ldots, P_{N}\right)=\sum_{v_{1}, \ldots, v_{N}} P\left(v_{1}, \ldots, v_{N}\right) \log \frac{P\left(v_{1}, \ldots, v_{N}\right)}{P\left(v_{1}\right) P\left(v_{2}\right) \cdots P\left(v_{N}\right)}
$$

This information measure is zero if the parameters are all independent of each other and its value increases as the dependence between the parameters increases. The maximum value is given by $N \log (r)$ where $r$ is the number of possible values of each parameter. Like the entropy measure in Eq. (28), we can compute this global information measure using local computations on the PDN. Let a parameter node, $P_{i}$, have parents denoted by $\Pi\left(P_{i}\right)$ with their values denoted by $\pi\left(P_{i}\right)$. Using the conditioned factored representation of the joint probability function, we can easily show that

$$
I\left(P_{1}, \ldots, P_{N}\right)=\sum_{i}\left(\sum_{v_{i}, \pi\left(P_{i}\right)} P\left(v_{i}, \pi\left(P_{i}\right)\right) \log \frac{P\left(v_{i} \mid \pi\left(P_{i}\right)\right)}{P\left(v_{i}\right)}\right) .
$$

As before (Eq. (29)), the outside (first) sum on the right-hand side of Eq. (31) is over the parameter nodes of the PDN. The inside (second) sum is over the states of the neighboring nodes and can be computed locally at each node. The conditional probability $P\left(P_{i}=\right.$ $\left.v_{i} \mid \Pi\left(P_{I}\right)=\pi\left(P_{i}\right)\right)$ is present, a priori, at the node and the local joint probability $P\left(P_{i}=\right.$ $\left.v_{i}, \Pi\left(P_{I}\right)=\pi\left(P_{i}\right)\right)$ can be estimated by locally accumulating count during Gibbs sampling. Thus, the mutual information-based dependence measure can be constructed by local computations on the PDN; this is, of course, computationally more efficient than summing over a potentially large joint probability space.

\section{RESULTS}

In this section we provide an empirical demonstration of the process of construction and performance of the PDN using (i) a small vision modules (Canny edge detector) with 4 parameters, (ii) a coupling of two vision modules (Etemadi et al.'s grouper [32]) with 7 parameters, and (iii) a combination of three vision modules (graph spectral-based complex grouper $[33,34]$ ) with 21 parameters. We allow for 10 possible choices of each parameter. As a test bed we consider an image set of 20 aerial images with hand-annotated ground truth showing the various object outlines. We divide these 20 images into sets $\left(S_{1}\right.$ and $\left.S_{2}\right)$ 
of 10 images each. Two study the performance of the PDN for each algorithm we train on one image set and test on the other.

We first review each of the three vision algorithms along with a discussion of the performance evaluation measures used for each. Second, we illustrate the PDN cnstruction process. Third, we discuss the performance of the PDN and the global nature of the parameter spaces using the information theoretic measures proposed in this paper.

\subsection{The Vision Subsystems and Evaluation Measures}

As an example of a simple vision module we use the Canny edge detector. The Canny edge detector has four parameters that need to be chosen: the scale, the edge length threshold, and the low and high hysteresis thresholds. We evaluate the performance of the edge detector using very simple signal-based criteria that have been suggested by many: the product of the true detection and percentage of correct response (i.e., one minus false alarm) rates. The ideal value of this feedback should be 1 , and zero indicates poor performance. The ground truth information consists of manual edge outlines of each object in the image. Note that we do not consider all intensity changes in the image but only those that belong to objects. The objects of interest in the aerial images are buildings, roads, hangars, and airplanes.

Etemadi et al.'s grouping module [32] is a good example of a vision subsystem that is a coupling of two modules, namely, an edge detection module and a grouping search module. The grouping module produces simple (2-ary or 3-ary) groupings of straight line segments such as parallels, continuous lines, T-junctions, $\mathrm{L}$-junctions, and $\lambda$-junctions. The total number of parameters are seven: three parameters are for the edge detector and four parameters specify the various grouping tolerances and their quality.

The graph spectra-based grouping algorithm described in [33, 34] is a good example of a complex vision subsystem that goes beyond the low level. The combination tries to form large groupings of constant curvature edge segments and consists of an edge detection module, a contour segmentation module, and a grouping module, which is based on the graph spectrum. We refer the reader to $[33,34]$ for details about the algorithm. Here we are concerned just with its parameter space behavior over the 21 parameters that can be chosen. There are 3 edge detection parameters, 3 contour segmentation parameters, 13 grouping tolerance parameters, and 2 graph spectral parameters.

To evaluate both the Etemadi and the spectral grouping algorithms, we use a subset of the recently proposed five measures [6]. The five measures capture various aspects of performance of grouping algorithms in the context of constrained search and indexing based object recognition schemes. In our present experiments, we use only the (three) measures that capture the performance of a grouping module in the context of a constrained searchbased recognition scenario.

Let a grouping algorithm produce $N^{g}$ number of groups of constant curvature segments and let the number of objects in the image be $N^{m}$. The ground truth consists of manually outlined boundaries of the objects in the image. The three used measures are defined as follows. The first two measures are computed for every pair of groups and objects with partial overlap and then averaged over the whole image. The third measure is computed over the whole image. We denote the number of features in a group by $s$ and those in an object by $m$.

1. First measure, $F_{s}^{c}$ : Let the number of features of a group that overlap with an object be denoted by $c_{g}$. Then, $F_{s}^{c}=c_{g} / s$. This fraction captures the speed of constrained 
search-based recognition of the object using the group. Let the average of this measure over the whole image be denoted by $\operatorname{Avg}\left(F_{s}^{c}\right)$.

2. Second measure, $F_{m}^{c}$ : Let the number of object features that overlap with a group be $c_{o}$. Then $F_{m}^{c}=c_{o} / m$. This captures the confidence in the constrained search-based recognition using the group. Let the average of this measure over the whole image be denoted by $\operatorname{Avg}\left(F_{m}^{c}\right)$.

3. Third measure, $F_{\text {true }}$ : This measure captures the fraction of the groups that are pure, $F_{\text {true }}=N_{\text {true }}^{g} / N^{g}$. Pure groups are groups that are completely contained inside an object and do not contain any spurious background features. The inverse of this measure is the average number of groups that need to be generated by a grouping algorithm for every pure group.

In the learning automata framework we need to combine these three measures to generate one feedback value that ranges from 0 to 1 . All the above measures range from 0 to 1 , with 1 being the optimal value. We consider the square root of the product of these three measures as the performance scalar, $\beta=\sqrt{\operatorname{Avg}\left(F_{s}^{c}\right) \operatorname{Avg}\left(F_{m}^{c}\right) F_{\text {true }}}$. The best case value of $\beta$ is 1 and corresponds to groups that cover every object completely without any spurious background feature and the false alarm rate is zero. Other combinations of the measures might be appropriate for different scenarios. The particular combination used here suffices for illustrative purposes.

\subsection{Constructing the PDN}

The first step of the PDN construction process is the stochastic sampling of the parameter spaces. Figure 9 shows the learning traces on image set $S_{1}$ for (a) the Etemadi and (b) the spectral grouping algorithm. The running average (over last 100 iterations) performance feedback are plotted against iteration. Note how the performance is enhanced with iteration. The learn rate for the Etemadi grouper was fixed at 0.1 . The parameter space of the Etemadi grouper is $\left(10^{14}\right.$ times) smaller than that of the spectral grouper; hence the convergence in Fig. 9a is faster than that seen in Fig. 9b. However, note that the convergence rate tends to scale up sublinearly with the parameter space size. Figure $9 \mathrm{~b}$, in addition to displaying the trace for a learn rate of $\mu=0.1$, shows traces for learn rates of 0.5 and 0.8 . As expected, the convergence rate is faster for larger learn rates, however, the performance at convergence is a bit lower than at slower learn rates. If time is a critical factor, then it might be possible to use a faster learn rate provided one is willing to accept reduction in performance. For the spectral grouping algorithm, which is the most complex of the three, each execution takes about $4 \mathrm{~s}$ on a Sparc-30 workstation. At each iteration, the grouping algorithm is run on the 10 images in a set, which translates to $40 \mathrm{~s}$ for each iteration. Thus, for $\mu=0.1$ (about 6000 iterations) it took about $67 \mathrm{~h}$. For $\mu=0.5$ (about 1500 iterations), it took $17 \mathrm{~h}$. These times are not prohibitive given that this parameter space sampling is an off-line process and is used to construct the PDN, which is intended to be used online.

Figure 10 shows the PDNs that are inferred for the three different algorithms: the edge detector, the Etemadi grouper, and the spectral grouper. The left column shows the PDNs that are learned on $S_{1}$ and the right column shows the PDNs that are learned on $S_{2}$. For the Etemadi and the spectral groupers, the PDNs on $S_{1}$ and $S_{2}$ do not share many common features. This is a consequence of two factors. First is the fact that we have imposed a polytree structure on the PDN and small perturbations in training data might result in different tree structures depending on the strengths of the dependencies. Multiple tree structures may be equally good approximations. This brings us to the second factor, namely, the low mutual 


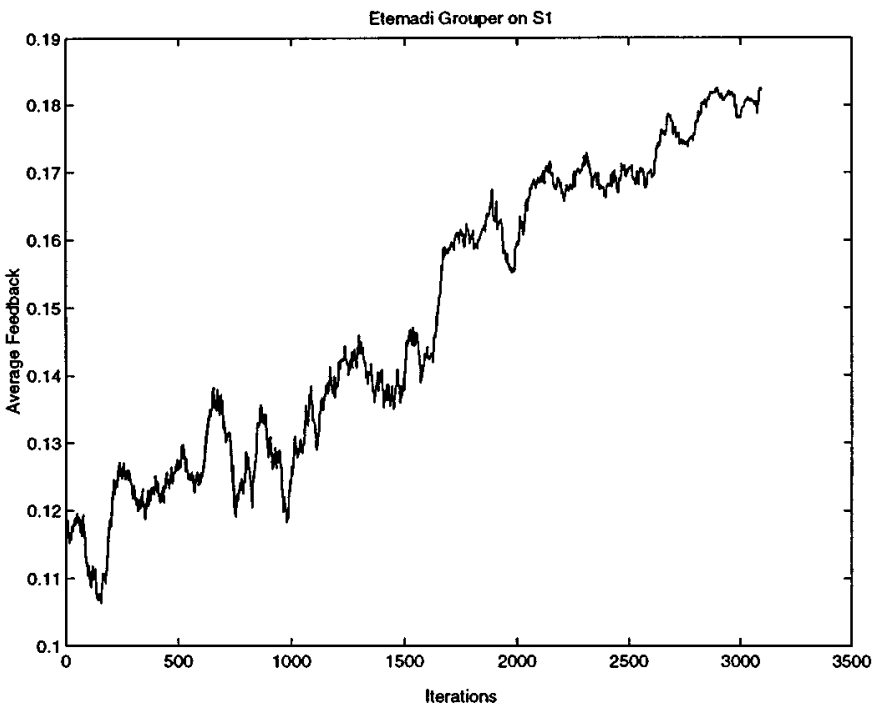

(a)

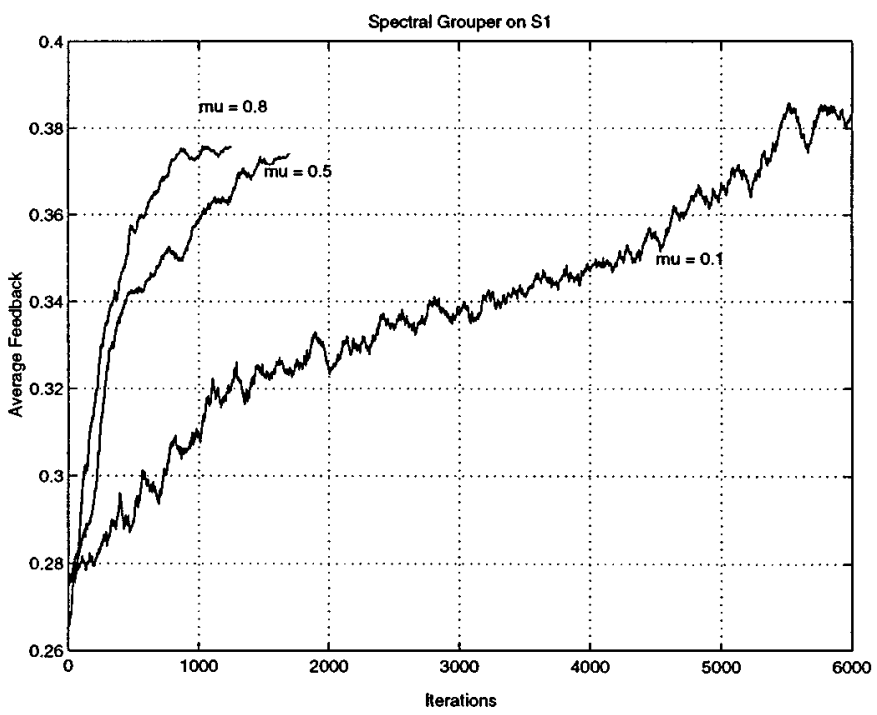

(b)

FIG. 9. Learning traces on image set $S_{1}$ for (a) the Etemadi grouping algorithm and (b) the graph spectral grouping algorithm. In (b) we show three traces corresponding to three different learning rates. The vertical axes represent the running average feedback over the last 100 iterations.

dependencies between the parameters. Except for a few links, most links had low mutual dependencies; thus, the strength of the links of the PDN is low. The total mutual information in the tree links (sum of all terms like Eq. (13), $\sum_{i j} I\left(P_{i}, P_{j}\right)$ ) is listed below each diagram in Fig. 10. Note that before comparing the sums we need to normalize them by the number of links in each PDN. This observation about the low mutual dependencies between the parameter (for the present set of vision modules) is borne out further by the global measures on the PDN and explicit experiments discussed in Section 7.4. 


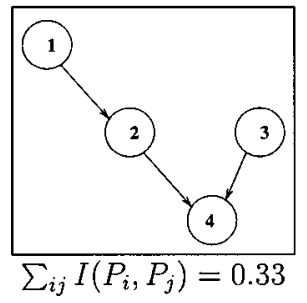

(a)

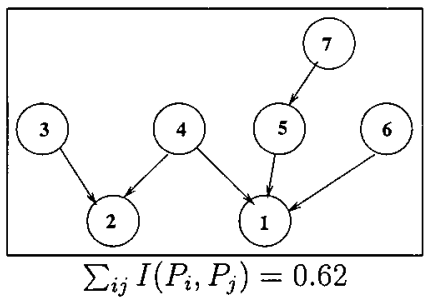

(c)

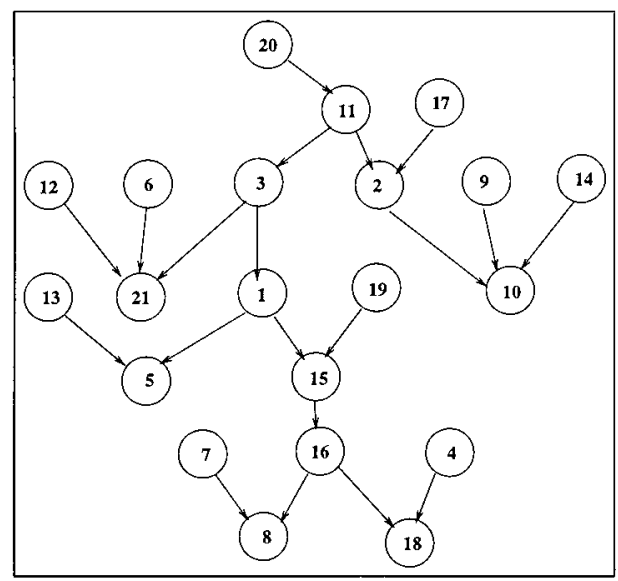

$\sum_{i j} I\left(P_{i}, P_{j}\right)=3.40$

(e)

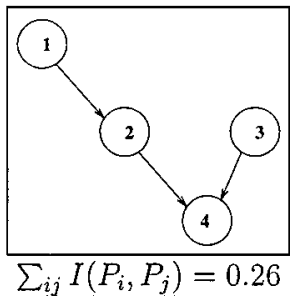

(b)

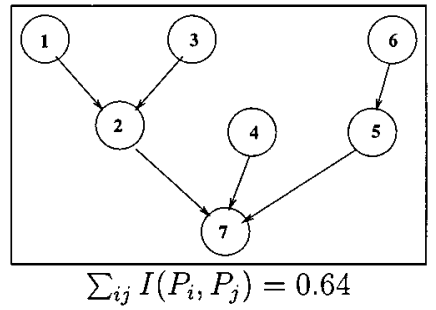

(d)

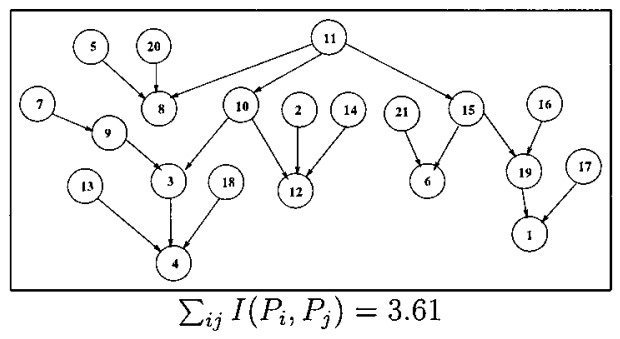

(f)

FIG. 10. Parameter dependence networks inferred for (a) the edge detector by training on $S_{1}$, (b) the edge detector by training on $S_{2}$, (c) the Etemadi grouper by training on $S_{1}$, (d) the Etemadi grouper by training on $S_{2}$, (e) the graph spectra-based grouper by training on $S_{1}$, and (f) the graph spectra-based grouper by training on $S_{2}$. The numbers below each diagram show the total mutual information over the tree links.

\subsection{Effectiveness of the PDN}

To study the effectiveness of the PDN, we explore its ability to generate good parameter sets. We ask: (i) Is the PDN able to generate better parameter sets than, say, random sampling? and (ii) How good are the PDN-generated parameters for images that are not part of the training set. Of course, the answer to the second question depends on the PDN and the extent of dependence of the particular algorithm on images. So in some instances, we might see a performance drop, but hopefully not a significant one.

As mentioned before, we consider a test bed of 20 images that we divide into two sets, $S_{1}$ and $S_{2}$, with 10 images in each. Figures 11, 12, and 13 show the performance spreads 


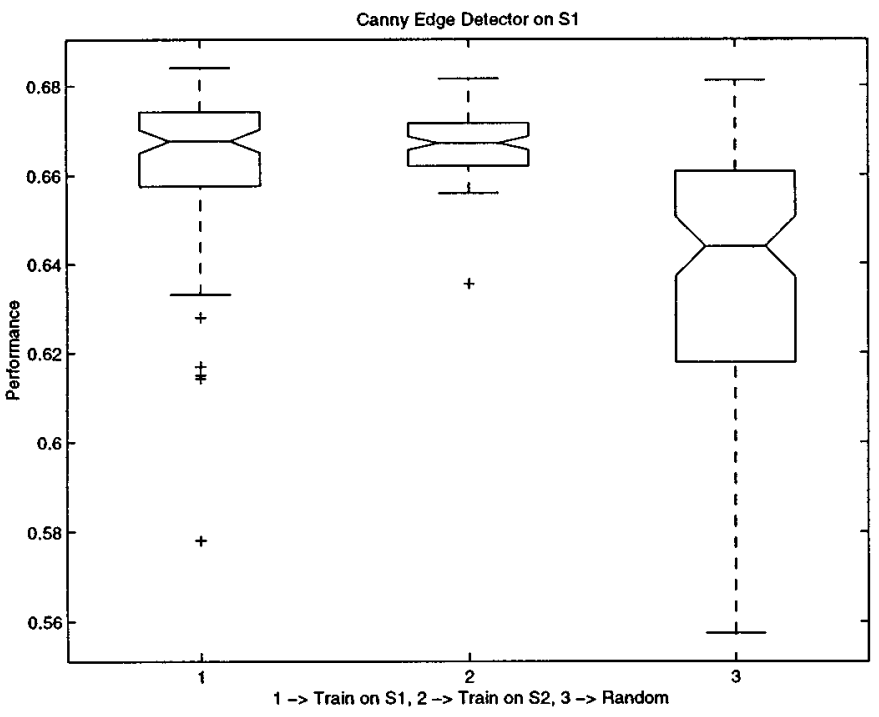

(a)

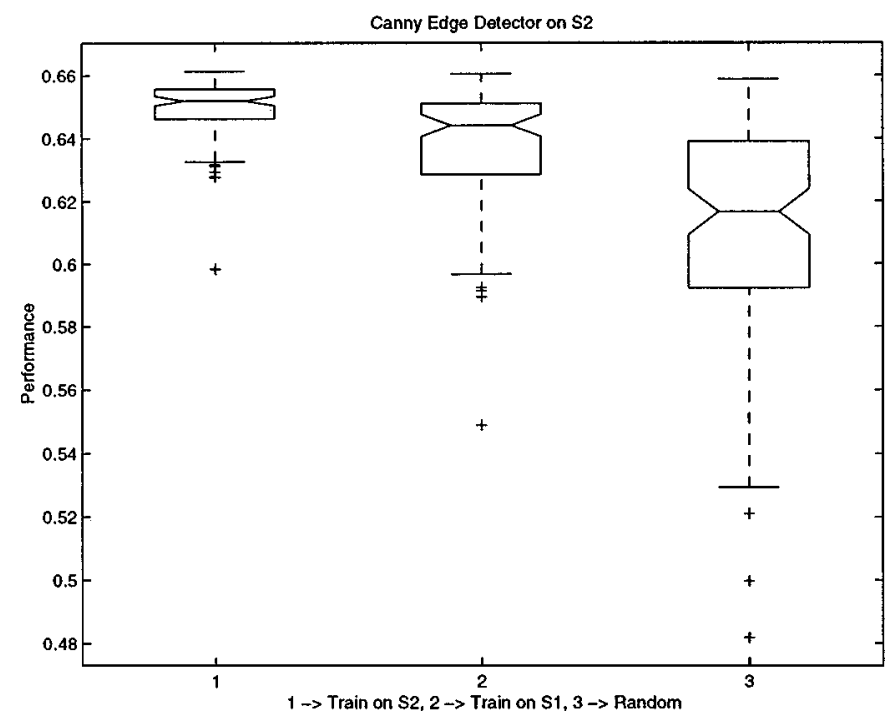

(b)

FIG. 11. Distribution of performance measures for the Canny edge detector: Results of tests on (a) set $S_{1}$ and (b) set $S_{2}$.

for a set of train and test experiments for the Canny edge detector, the Etemadi grouper, and the spectral grouper, respectively. The box-and-whisker plots in each figure depict the performance on the set under consideration, $S_{1}$ or $S_{2}$. Each box has lines at the lower quartile, median, and upper quartile values. The whiskers are lines extending from each end of the box to show the extent of the rest of the data. Outliers are data with values beyond the ends of the whiskers and are marked with pluses.

Figures 11a, 12a, and 13a plot the performance spread on image set $S_{1}$. The leftmost box-and-whisker plots in each of these figures show the performance spread with 100 parameter sets generated with a PDN constructed by training on set $S_{1}$ itself, the middle 


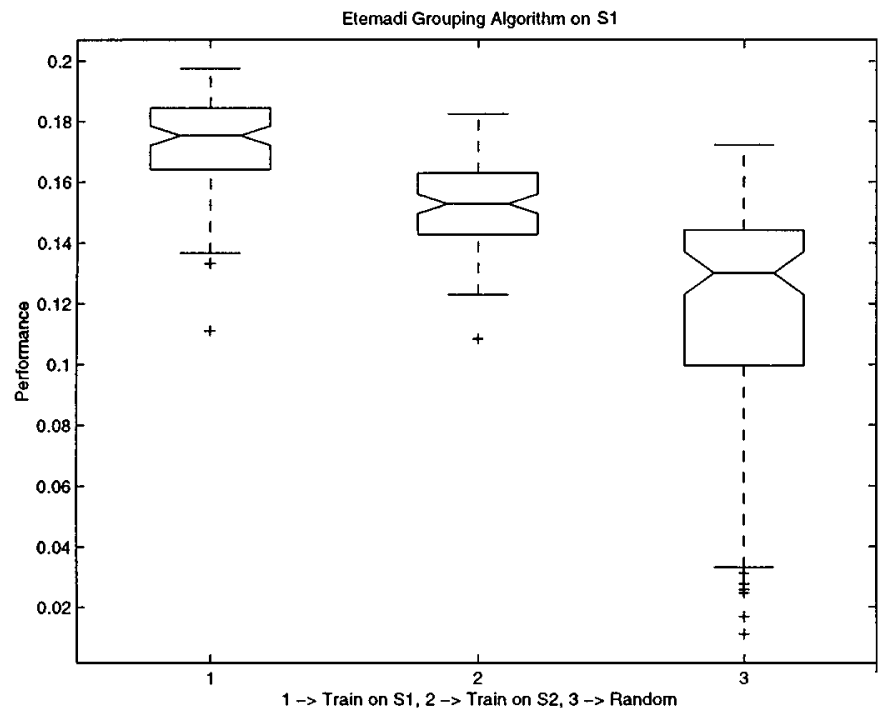

(a)

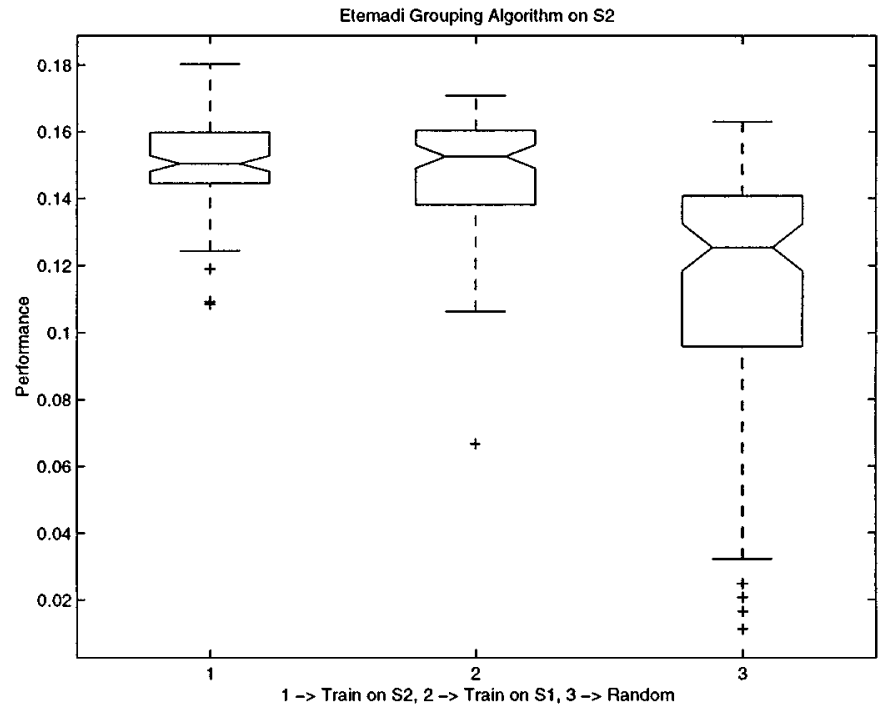

(b)

FIG. 12. Distribution of performance measures for the Etemadi et al. grouping module: Results of tests on (a) set $S_{1}$ and (b) set $S_{2}$.

box-and-whisker plots are with 100 parameters generated with a PDN constructed by training on set $S_{2}$, and the rightmost box-and-whisker plots are with 100 parameter sets generated (uniform) randomly. The PDN-based parameters were generated by Gibbs sampling as described in Section 6.1. Similarly, Figs. 11b, 12b, and 13b show the test results on image set $S_{2}$. Note how the performance with training and testing on different sets (middle box-and-whisker plots) is close to the performance obtained by training and testing on the same set (leftmost box-and-whisker plots). As expected, the spread with random parameter sets has more variation than the ones generated with the PDN, thus demonstrating 


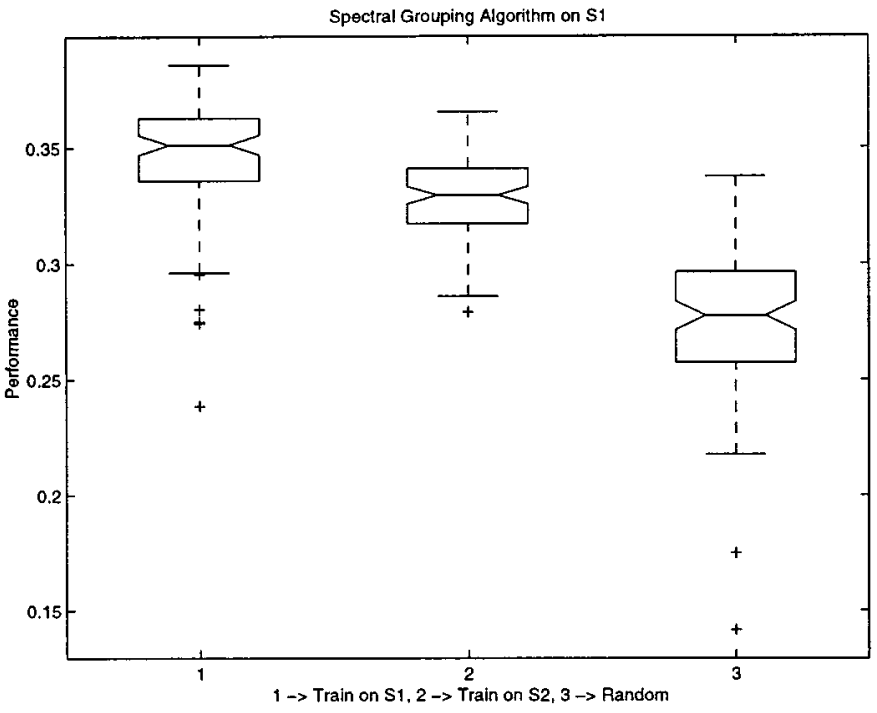

(a)

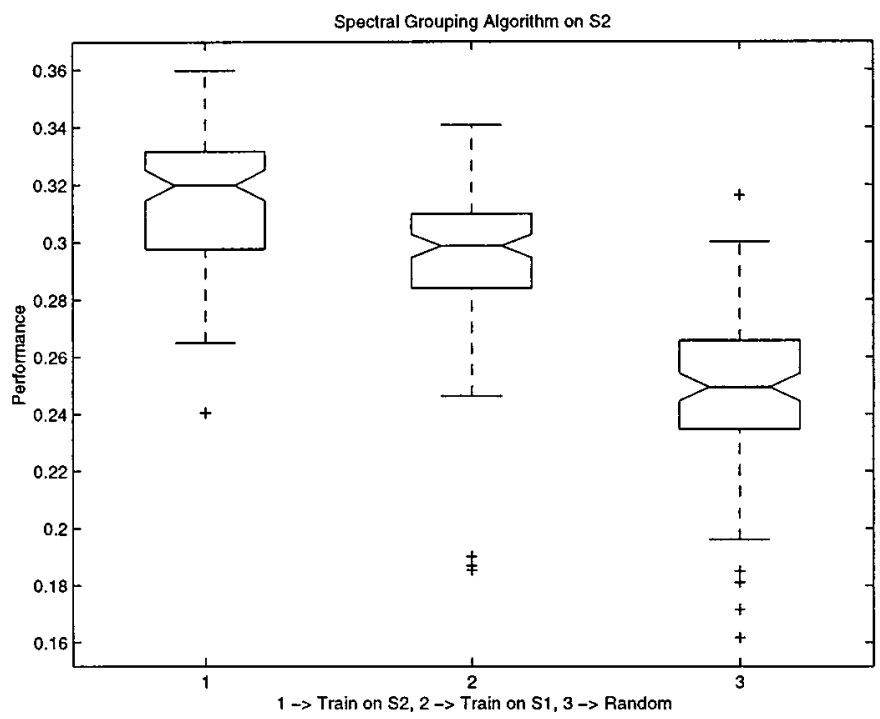

(b)

FIG. 13. Distribution of performance measures for the graph spectral-based perceptual grouping module: Results of tests on (a) set $S_{1}$ and (b) set $S_{2}$.

that the PDNs are able to generate good parameter sets. The observation that the train and test experiments on different image sets and on the same image sets overlap significantly supports the utility of the PDN beyond the training set of images. Of course, the amount of performance overlap depends on the particular algorithm. We discuss this effect next.

\subsection{What Can We Infer from the PDN?}

Table 3 lists the values of the global sensitivity and interparameter dependence measures for the three algorithms that were derived from the PDN. The first row of numbers lists the parameter sensitivities, $S\left(P_{1}, \ldots, P_{N}\right)$ (Eq. 28), on each image set. The smaller the value 
TABLE 3

Global Sensitivity and Interparameter Dependence Measures for the Canny Edge Detector, the Etemadi Grouper, and the Spectral Grouper

\begin{tabular}{lrrrrrrrrr}
\hline \multirow{2}{*}{ Measures } & \multicolumn{2}{c}{ Spectral } & & \multicolumn{2}{c}{ Etemadi } & & \multicolumn{2}{c}{ Canny edge } \\
\cline { 2 - 3 } & $S_{1}$ & $S_{2}$ & & $S_{1}$ & $S_{2}$ & & $S_{1}$ & $S_{2}$ \\
\hline Sensitivity & 31.3 & 34.2 & & 14.6 & 17.1 & & 11.3 & 10.8 \\
max. possible & 69.8 & 69.8 & & 23.3 & 23.3 & & 13.3 & 13.3 \\
Dependence & 3.7 & 3.4 & & 1.2 & 2.1 & & 0.7 & 0.5 \\
\hline
\end{tabular}

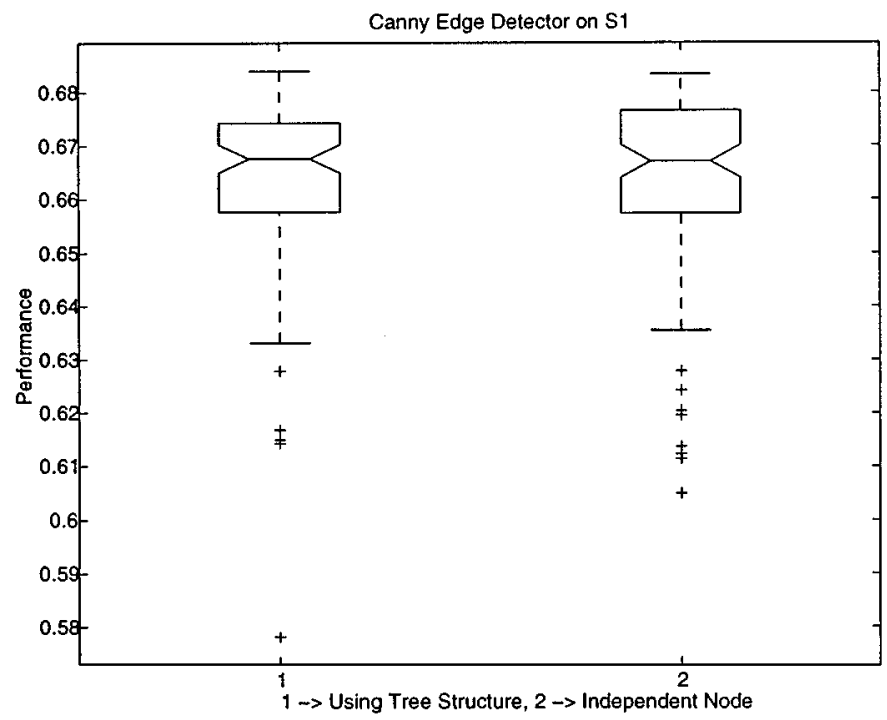

(a)

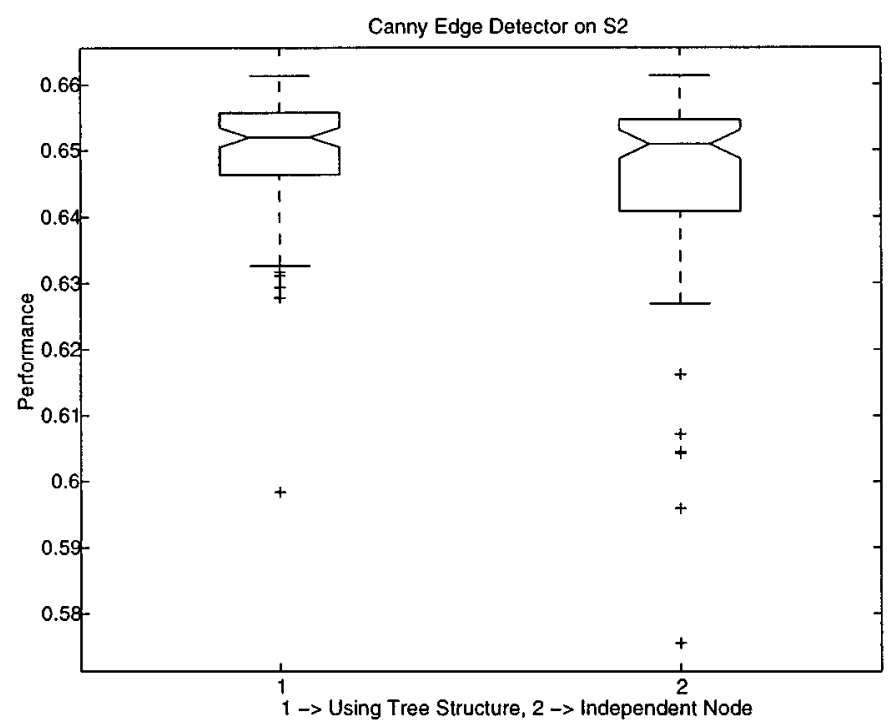

(b)

FIG. 14. Distribution of performance measures for the Canny edge detector for a polytree PDN versus a PDN with no links on (a) set $S_{1}$ and (b) set $S_{2}$. The ANOVA significances ( $p$-values) of the differences seen are as follows: (a) 0.9389 (not significant difference), (b) 0.0364 (significant difference). 


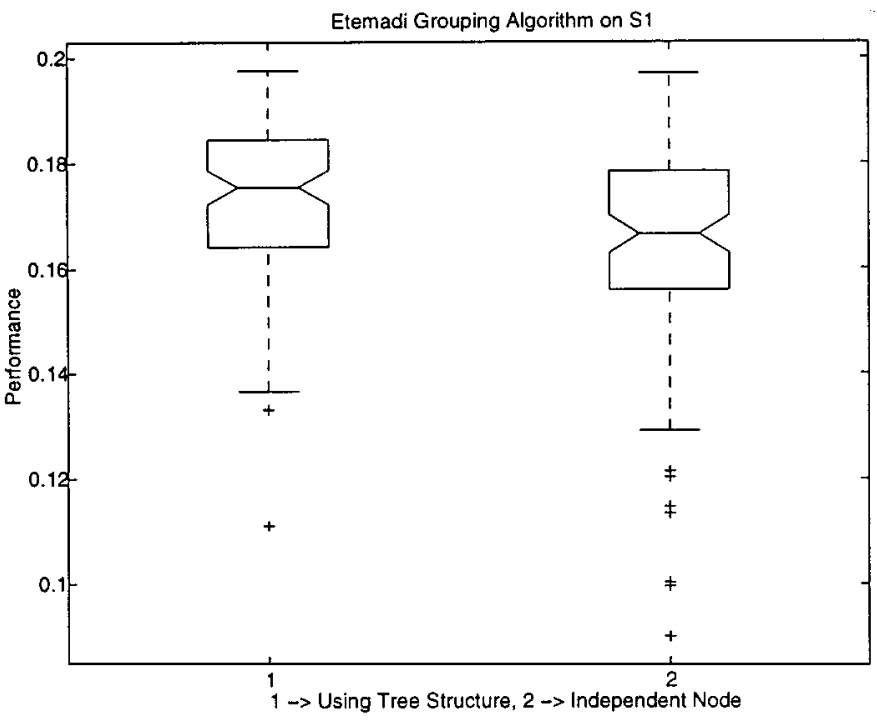

(a)

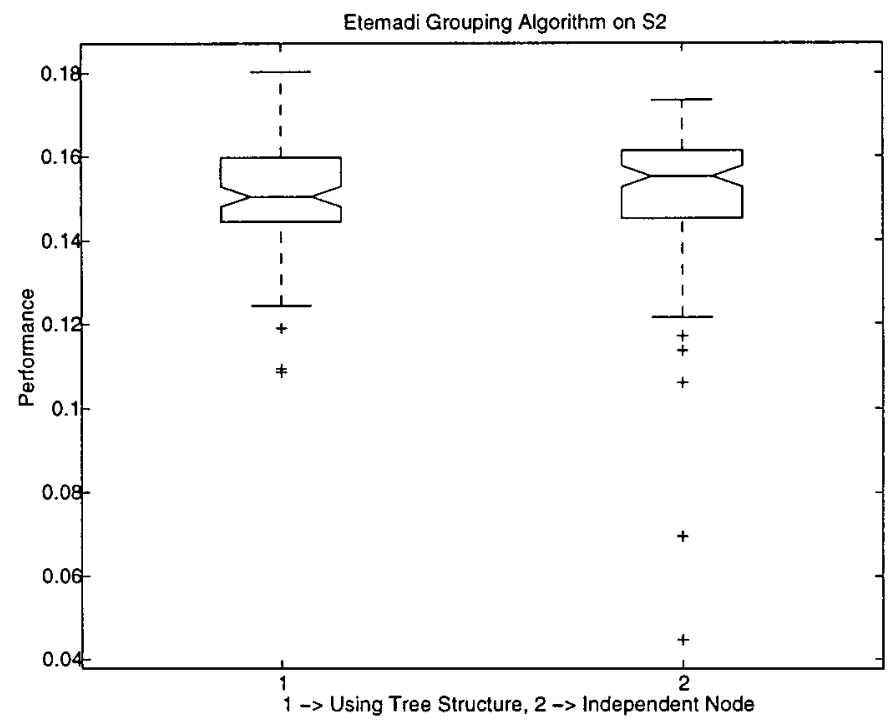

(b)

FIG. 15. Distribution of performance measures for the Etemadi grouper for a polytree PDN versus a PDN with no links on (a) set $S_{1}$ and (b) set $S_{2}$. The ANOVA significances ( $p$-values) of the differences seen are as follows: (a) 0.0001 (significant difference), (b) 0.7100 (not significant difference).

of $S\left(P_{1}, \ldots, P_{N}\right)$, the larger is the dependence of the algorithm performance on the proper choice of the parameters. For algorithms with large values of $S\left(P_{1}, \ldots, P_{N}\right)$, there is less need for parameter tuning. The maximum possible value for $S\left(P_{1}, \ldots, P_{N}\right)$ depends on the size of the parameter space and is reached when all parameter combinations result in the same performance. Thus, for a 21 parameter space with each parameter taking on 10 possible values, the maximum value of $S\left(P_{1}, \ldots, P_{N}\right)$ is $\log \left(10^{21}\right)=69.8$. These maximum possible values are listed as the second row of numbers in Table 3. On comparing the 


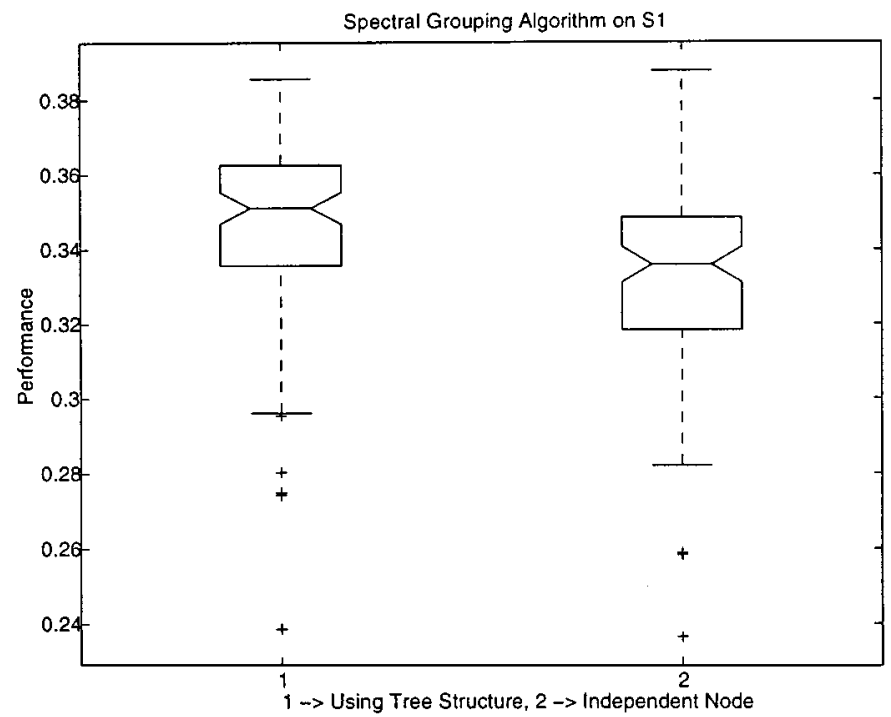

(a)

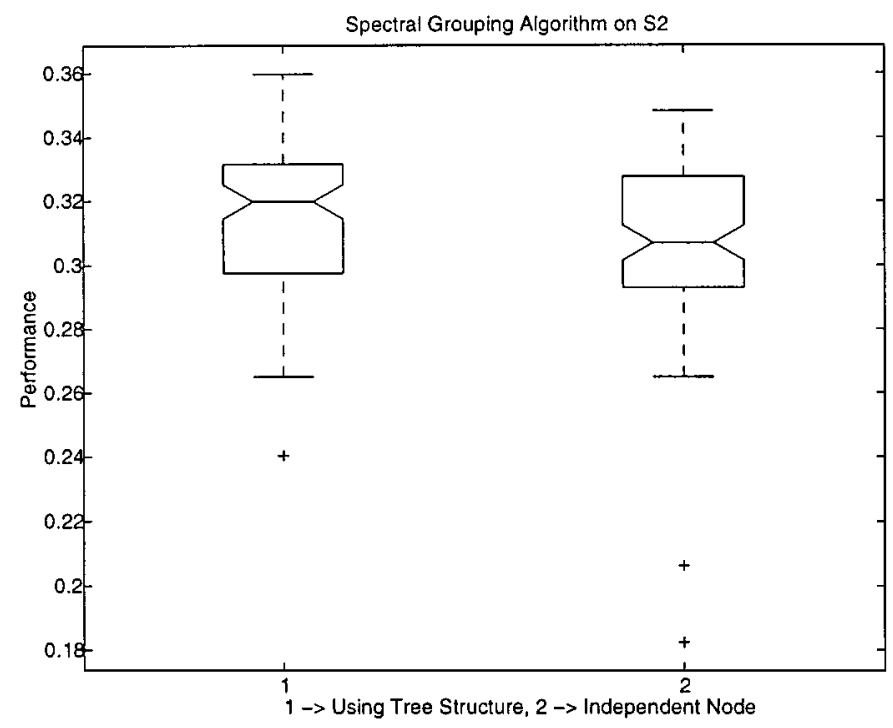

(b)

FIG. 16. Distribution of performance measures for the spectral grouper for a polytree PDN versus a PDN with no links on (a) set $S_{1}$ and (b) set $S_{2}$. The ANOVA significances ( $p$-values) of the differences seen are as follows: (a) 0.015 (significant difference), and (b) 0.0170 (significant difference).

sensitivities of each algorithm to the maximum possible we see that the Canny edge detector is the least sensitive of the three and the spectral grouper is the most sensitive of the three algorithms. This observation correlates well with the drop in performance on training and testing on different image sets that we presented in Figs. 11, 12, and 13. Also note that for the spectral-based grouper, the performance with random parameter sets is significantly lower than the trained ones (Fig. 13). This suggests that the PDN is most appropriate for algorithms that have low values of $S\left(P_{1}, \ldots, P_{N}\right)$. For complex algorithms involving many 
modules we expect the performance to be strongly linked with the proper choice of the parameters and the utility of the PDN would be enhanced in such cases.

The third row of numbers in Table 3 are the interparameter dependence measures, $I\left(P_{1}, \ldots, P_{N}\right)$, (Eq. (30)) for the three algorithms. A value of zero suggests complete independence of parameters. The larger the value for this measure, the greater is the interparameter dependence. We note that for all the three algorithms this value is low, which is important since it implies that each parameter can be tuned independent of another. This lack of overall strong dependence also explains the lack of stability of the inferred PDN structures (Fig. 10). Of course, this observation is only for the vision modules that we studied.

We verified this observation of low parameter interdependence experimentally. For each of the three vision algorithms, we compared the performance of a polytree PDN with a PDN with no links, i.e., independent nodes, on each of the two images sets $S_{1}$ and $S_{2}$. We learned these two PDN structures and tested them on the same image set, so as to eliminate learnability issues and to highlight parameter space modeling issues. Learning a PDN with no links is easy, since the structure is already specified and the probabilities are just the individual node probabilities, which are estimated as described in Section 5.2. Figures 1416 show the training performance of a learned polytree PDN versus learned independent node PDN for all three vision algorithms studied in this paper. In general, there is slight performance degradation on using a PDN with no links: the median performance is lower and/or the spread is wider for a PDN with no links. However, as noted in the figure, the performance differences are not significant for the Canny edge detector and the Etemadi grouping algorithm on one of the image sets. The differences are significant on both the image sets for the spectral grouping algorithm.

\section{CONCLUSIONS}

In this paper we proposed a novel Bayesian network-based formalism, which we call the parameter dependence networks, to model the parameter spaces of vision systems. The PDNs abstract the behavior of parameter spaces using probabilistic measures and explicitly model the interparameter dependence. We provide a technique to learn these PDNs from stochastic samples of the parameter spaces, obtained by a team of learning automata. We demonstrate the construction of the PDN for real algorithms with a number of parameters ranging from 4 to 21 .

The PDNs are useful in suggesting good parameter sets, which is particularly important in online parameter tuning of closed-loop vision systems and in performance evaluation of vision algorithms. The PDNs are especially useful in choosing parameters under the constraint that certain parameter values need to be fixed to a particular value. We can also gain insights into the nature of the parameter space by defining global measures of parameter sensitivity and interdependence based on the concepts of entropy and mutual information.

\section{REFERENCES}

1. V. Murino, G. L. Foresti, and C. S. Regazzonni, A distributed probabilistic system for adaptive regulation of image processing parameters, IEEE Trans. Systems, Man, and Cybernetics, B 26, 1996, 1-20.

2. J. Peng and B. Bhanu, Closed loop object recognition using reinforcement learning, IEEE Trans. Pattern Anal. Mach. Intelligence 20, 1998, 139-154. 
3. V. Ramesh, R. M. Haralick, X. Zhang, D. C. Nadadur, and K. Thornton, Automatic selection of tuning parameters for feature extraction sequences, in Proceedings of the Conference on Computer Vision and Pattern Recognition, pp. 672-677, 1994.

4. H. I. Christensen and W. Forstner, Performance characteristics of vision algorithms, Mach. Vision Appl. 9, 1997, 215-218.

5. K. Cho, P. Meer, and J. Cabrera, Performance assesment through bootstrap, IEEE Trans. Pattern Anal. Mach. Intelligence 19, 1997, 1185-1198.

6. S. Borra and S. Sarkar, A framework for performance characterization of intermediate-level grouping modules, IEEE Trans. Pattern Anal. Mach. Intelligence 19, 1997, 1306-1312.

7. M. Heath, S. Sarkar, T. Sanocki, and K. W. Bowyer, A robust visual method for assessing the relative performance of edge-detection algorithm, IEEE Trans. Pattern Anal. Mach. Intelligence 19, 1997, 13381359.

8. J. L. Barron, D. J. Fleet, and S. S. Beauchemin, Performance of optical flow techniques, Int. J. Comput. Vision 12, 1994, 43-77.

9. R. Zhang, P. S. Tsai, J. E. Cryer, and M. Shah, Analysis of shape from shading techniques, in Proceedings of the Conference on Computer Vision and Pattern Recognition, pp. 377-384, 1994.

10. V. Ramesh and R. M. Haralick, Random perturbation models and performance characterization in computer vision, in Proceedings of the Conference on Computer Vision and Pattern Recognition, pp. 521-527, 1992.

11. T. Kanungo, M. Y. Jaisimha, and R. M. Haralick, A methodology for quantitative performance evaluation of detection algorithms, IEEE Trans. Image Process. 4, 1995, 1667-1674.

12. S. Dougherty and K. W. Bowyer, A formal framework for the objective evaluation of edge detectors, in Proceedings of the International Conference on Image Processing, Oct. 1998.

13. S. Houzelle, T. M. Strat, P. Fua, and M. A. Fischler, Using contextual information to set control parameter of a vision process, in Proceedings of the International Conference on Pattern Recognition, A, pp. 830-832, 1994.

14. V. Murino, G. L. Foresti, and C. S. Regazzoni, A belief based approach for adaptive image processing, Int. J. Pattern Recognition Artificial Intelligence 11, 1997, 359-392.

15. S. Z. Li, Parameter estimation for optimal object recognition: Theory and application, Int. J. Comput. Vision 21, 1997, 207-222.

16. M. Pelillo and M. Refice, Learning compatibility coefficients for relaxation labelling processes, IEEE Trans. Pattern Anal. Mach. Intelligence 16, 1994, 933-945.

17. J. Pearl, Probabilistic Reasoning in Intelligent Systems, Morgan Kaufman, San Mateo, CA, 1988.

18. G. F. Cooper, The computational complexity of probabilistic inference using Bayesian belief networks, Artificial Intelligence 42, 1990, 393-405.

19. K. S. Narendra and M. L. Thathachar, Learning Automata: An Introduction, Prentice Hall, New York, 1989.

20. M. L. Thathachar and P. S. Sastry, Learning optimal discriminant functions through a cooperative game of automata, IEEE Trans. Systems, Man, and Cybernetics 17, 1987, 73-85.

21. W. Buntine, A guide to the literature on learning probabilistic networks from data, IEEE Trans. Knowledge Data Engineering 8, 1996, 195-210.

22. E. H. Herskovits and G. F. Cooper, Kutato: An entropy driven system for construction of probabilistic expert systems from databases, in Proceedings of the 6th Conference on Uncertainty in Artificial Intelligence, pp. 54-62, 1990.

23. C. K. Chow and C. N. Liu, Approximating discrete probability distributions with dependence trees, IEEE Trans. Inform. Theory 14, 1968, 462-467.

24. W. Lam and F. Bacchus, Using causal information and local measures to learn Bayesian networks, in Proceedings of the 9th Conference on Uncertainty in Artificial Intelligence, pp. 243-250, 1993.

25. A. E. Raftery, Bayesian model selection in social research, in Sociological Methodology (P. V. Marsden, Ed.), Blackwells, Cambridge, MA, 1995.

26. D. Heckerman, D. Geiger, and D. Chickering, "Learning Bayesian Networks: The Combination of Knowledge and Statistical Data," Tech. Rep. MSR-TR-94-09, Microsoft Research, 1994.

27. R. E. Kass and A. E. Raftery, Bayes factor and model uncertainty, J. Am. Statist. Assoc. 90, 1995, 773-795. 
28. D. J. Spiegelhalter and S. L. Lauritzen, Sequential updating of conditional probabilities on directed graphical structures, Networks 20, 1990, 579-605.

29. G. Rebane and J. Pearl, The recovery of causal poly-tree from statistical data, in Proceedings of the 3rd Workshop on Uncertainty in Artificial Intelligence, pp. 222-228, 1987.

30. S. Abe, Expected relative entropy between a finite distribution and its empirical distribution, SUT J. Math. 32, 1996, 149-156.

31. C. S. Jensen, A. Kong, and U. Kjaerulff, Blocking Gibbs Sampling in Very Large Probabilistic Expert Systems, Tech. Rep. R-93-2031, Institute for Electronic Systems, Aalborg, Denmark, Oct. 1993.

32. A. Etemadi, J.-P. Schmidt, G. Matas, J. Illingworth, and J. Kittler, Low-level grouping of straight line segments, in Proceedings of the British Machine Vision Conference, pp. 119-126, Springer-Verlag, Berlin/New York, 1991.

33. S. Sarkar, Learning to form large groupings of salient image features, in Proceedings of the Conference on Computer Vision and Pattern Recognition, pp. 780-786, 1998.

34. S. Sarkar and P. Soundararajan, Supervised learning of large perceptual organization: Graph spectral partitioning and learning automata, to appear in IEEE Trans. Pattern Anal. Mach. Intelligence, 2000. 